FTPI-MINN-04/07, UMN-TH-2234/04

ITEP-TH-12/04

\title{
Non-Abelian String Junctions as Confined Monopoles
}

\author{
M. Shifman ${ }^{a}$ and A. Yung ${ }^{a, b, c}$ \\ ${ }^{a}$ William I. Fine Theoretical Physics Institute, University of Minnesota, \\ Minneapolis, MN 55455, USA \\ ${ }^{b}$ Petersburg Nuclear Physics Institute, Gatchina, St. Petersburg 188300, Russia \\ ${ }^{c}$ Institute of Theoretical and Experimental Physics, Moscow 117250, Russia
}

\begin{abstract}
Various dynamical regimes associated with confined monopoles in the Higgs phase of $\mathcal{N}=2$ two-flavor QCD are studied. The microscopic model we deal with has the $\mathrm{SU}(2) \times \mathrm{U}(1)$ gauge group, with a Fayet-Iliopoulos term of the $\mathrm{U}(1)$ factor, and large and (nearly) degenerate mass terms of the matter hypermultiplets. We present a complete quasiclassical treatment of the BPS sector of this model, including the full set of the first-order equations, derivations of all relevant zero modes, and derivation of an effective low-energy theory for the corresponding collective coordinates. The macroscopic description is provided by a $C P^{1}$ model with or without twisted mass. The confined monopoles - string junctions of the microscopic theory - are mapped onto BPS kinks of the $C P^{1}$ model. The string junction is $1 / 4$ BPS. Masses and other characteristics of the confined monopoles are matched with those of the $C P^{1}$-model kinks. The matching demonstrates the occurrence of an anomaly in the monopole central charge in $4 \mathrm{D}$ Yang-Mills theory. We study what becomes of the confined monopole in the bona fide non-Abelian limit of degenerate mass terms where a global $\mathrm{SU}(2)$ symmetry is restored. The solution of the macroscopic model is known e.g. from the mirror description of the $C P^{1}$ model. The monopoles, aka $C P^{1}$-model kinks, are stabilized by nonperturbative dynamics of the $C P^{1}$ model. We explain an earlier rather puzzling observation of a correspondence between the BPS kink spectrum in the $C P^{1}$ model and the Seiberg-Witten solution.
\end{abstract}




\section{Contents}

1 Introduction $\quad 2$

$\begin{array}{lll}2 & \text { A brief summary of the theoretical set-up } & 7\end{array}$

3 Non-Abelian strings $\quad 9$

3.1 Abrikosov-Nielsen-Olesen vs. elementary strings . . . . . . . . . . . . 11

3.2 Embedding; first-order equations for the elementary strings . . . . . . . . . . . . . . . . . . . 13

3.3 Non-Abelian moduli . . . . . . . . . . . . . . . . . . 15

3.4 Supersymmetry . . . . . . . . . . . . . . . . . . . 19

4 Macroscopic theory $\quad 20$

4.1 Deriving the kinetic term; a basic normalizing integral . . . . . . . . 20

4.2 (1+1)-dimensional $C P^{1}$ model . . . . . . . . . . . . . . 23

4.3 Unequal quark mass terms; $C P^{1}$ with the twisted mass . . . . . . . . 26

4.4 Fermion zero modes of the string . . . . . . . . . . . . . . 31

5 Sigma-model kinks: monopoles of the microscopic theory 35

5.1 Microscopic theory: first-order master equations . . . . . . . . . . . . 36

5.2 The string junction solution for $\sqrt{\xi} \gg \mu \gg \Lambda$. . . . . . . . . . 38

5.3 More on kinks in the $C P^{1}$ model with the twisted mass . . . . . . . . 42

5.3.1 The $\theta$ term . . . . . . . . . . . . . . . . . . . 42

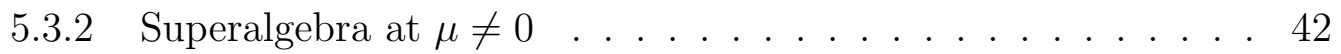

5.3.3 Kinks in the quasiclassical limit . . . . . . . . . . . . . . 43

5.3.4 Quantization of the bosonic moduli . . . . . . . . . . . 43

5.3.5 Switching on fermion moduli . . . . . . . . . . . . . 45

5.3 .6 Exact solution . . . . . . . . . . . . . . . . . 46

5.3.7 Multiplicity ................... 46

5.4 Implications for confined monopoles . . . . . . . . . . . . . . . 47

6 Anomaly: matching the central charges 48

7 2D sigma-model kink and 4D Seiberg-Witten exact solution $\quad 50$

8 Conclusions $\quad 53$

$\begin{array}{ll}\text { References } & 57\end{array}$ 


\section{Introduction}

This is the third work in the project devoted to investigation of the string/D-brane phenomena in supersymmetric gauge theories [1,2]. In the first work [1] we studied Abelian strings ending on BPS domain walls (D-branes) and localization of an (2+1)dimensional U(1) gauge field on the wall. The second work [2] was devoted to localization of non-Abelian fields on a stack of BPS domain walls, and 1/4 BPS junctions of non-Abelian strings with the walls. Here we extend the analysis and study $1 / 4$ BPS non-Abelian string junctions. This very interesting phenomenon has a clear-cut physical picture behind it - it describes monopoles in the confined phase. We are building the present analysis on our previous results, as well as on the results of Refs. [3, 4, 5, 6] interspersed in the fabric of the present work.

Results and techniques of string/D-brane theory, being applied to non-Abelian field theories (both, supersymmetric and non-supersymmetric), lead to qualitative and quantitative predictions which became especially numerous after the discovery [7] of the ADS/CFT correspondence. If gauge theories at strong coupling are in a sense dual to string/D-brane theory, they must support domain walls (or D-branes) [8], and we know, they do $[9,10]$. In addition, string/D-brane theory teaches us that a fundamental string that starts on a confined quark, can end on the domain wall. In the dual description the confined quark becomes a confined monopole. This is our primary object of study in the present paper. The question we ask is how the confined monopole connects to the flux tube.

Our task is to study this phenomenon in a controllable manner. To this end we will use a unique model which emerged recently as the one providing an ideal theoretical environment. The model will allows us to study, in a quasiclassical regime, confined monopoles whose magnetic flux flows through non-Abelian strings attached to them. The predictive power of the model derives from the fact that it has exact $\mathcal{N}=2$ supersymmetry (i.e. eight supercharges). The non-Abelian strings are 1/2 BPS, while the confined monopoles are 1/4 BPS saturated.

Let us outline some basic features of this model, which we will refer to as "microscopic." We consider $\mathcal{N}=2$ QCD $[11,12]$ with the gauge group $\mathrm{SU}(2) \times \mathrm{U}(1)$ with $N_{f}=2$ flavors of massive fundamental matter hypermultiplets (quarks) perturbed by the Fayet-Iliopoulos (FI) term [13] of the U(1) factor. In this theory we focus on a special so-called $r=2$ vacuum $[14,15,16]$ in which two quark flavors develop vacuum expectation values (VEV's). This vacuum is at weak coupling if the quark mass terms $m_{1,2}$ are large enough. They may or may not be equal. We consider both cases. If $m_{1}=m_{2}$ the $\mathrm{SU}(2)$ gauge group remains unbroken by VEV's of the adjoint fields. The quark condensation does break the gauge group $\mathrm{SU}(2) \times \mathrm{U}(1)$ at a scale $\xi$ (FI parameter) but leaves a global diagonal $\mathrm{SU}(2)_{C+F}$ subgroup of the gauge and flavor groups unbroken. It was recently shown [3] that in this case the flux tubes (strings) acquire additional orientational zero modes associated with rotation 
of the color magnetic flux inside the $\mathrm{SU}(2)$ group (similar results in three dimensions were obtained in [5]). This makes them genuinely non-Abelian. Moreover, it was found $[3,5]$ that the low-energy dynamics of the orientational zero modes of the non-Abelian strings are described by an effective $(1+1)$-dimensional $C P^{1}$ model ${ }^{1}$ on the string world sheet. The two-dimensional $C P^{1}$ model describing dynamics of the collective coordinates will be referred to as "macroscopic."

In fact, we can view our $\mathrm{SU}(2) \times \mathrm{U}(1)$ microscopic theory as a theory with the gauge group $\mathrm{SU}(3)$ broken down to $\mathrm{SU}(2) \times \mathrm{U}(1)$ at a large scale, of the order of $m_{1,2}$. This $\mathrm{SU}(3)$ theory has three types of monopoles associated with three roots of $\mathrm{SU}(3)$ algebra. Two of them are confined by "elementary" strings which we denote as $(1,0)$ and $(0,1)[16]$ (here $(n, k)$ denotes the string with two winding numbers $n$ and $k$ with respect to two U(1) subgroups of SU(3), see Sect. 3.1 for more details). These monopoles are very heavy, with masses of the order of $m_{1,2} / g^{2}$, and we do not touch them in this paper. They were considered recently in Ref. [17]. We will study only the monopoles which lie entirely inside the $\mathrm{SU}(2)$ factor of the $\mathrm{SU}(3)$ "proto" group. They are much lighter than $m_{1,2} / g^{2}$. Classically, on the Coulomb branch (i.e. when the FI parameter $\xi$ vanishes), their mass is proportional to $|\Delta m| / g^{2}$ where $\Delta m=$ $m_{1}-m_{2}$. In the limit $\Delta m \rightarrow 0$ they become massless, formally, in the classical approximation. Simultaneously their size become infinite [18]. The mass and size are stabilized by confinement effects which are highly quantum. The confinement of monopoles occurs on the Higgs branch, at $\xi \neq 0$. An interplay between $\Delta m, \xi$ and the dynamical Yang-Mills scale $\Lambda$ leads $^{2}$ to a spectrum of dynamical scenarios, all of which are interesting and will be discussed in the present paper from a unified point of view.

A qualitative evolution of the monopoles under consideration as a function of the relevant parameters is presented in Fig. 1 . We begin with the limit $\xi \rightarrow 0$ while $\Delta m$ is kept fixed. Then the corresponding microscopic theory supports the conventional (unconfined) 't Hooft-Polyakov monopoles [19] due to the spontaneous breaking of the gauge $\mathrm{SU}(2)$ down to $\mathrm{U}(1)$,

$$
\left\langle a^{3}\right\rangle=-\frac{1}{\sqrt{2}} \Delta m
$$

(the upper left corner of Fig. 1). If we allow $\xi$ be non-vanishing but

$$
|\Delta m| \gg \sqrt{\xi}
$$

then the effect which comes into play first is the above spontaneous breaking of the gauge $\mathrm{SU}(2)$. Further gauge symmetry breaking, due to $\xi \neq 0$, which leads to complete Higgsing of the model and the string formation (confinement of monopoles)

\footnotetext{
${ }^{1} C P^{N-1}$ for the gauge group $\mathrm{SU}(N) \times \mathrm{U}(1)$.

${ }^{2} \Lambda$ is also the dynamical scale of the $2 \mathrm{D} C P^{1}$ model.
} 


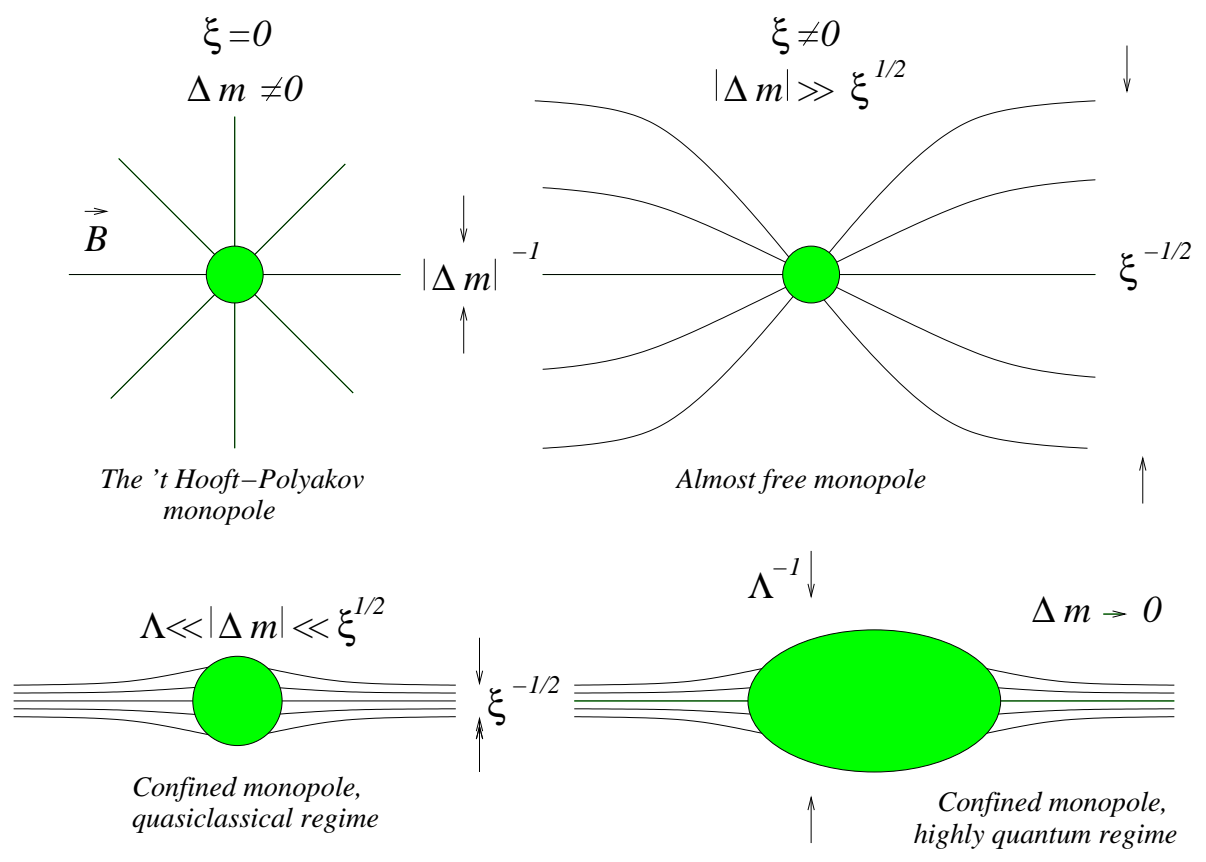

Figure 1: Various regimes for the monopoles and flux tubes. The dynamical scale parameters $\Lambda$ are the same in the microscopic and microscopic theories. The effective world-sheet theory we derive - the twisted-mass $C P^{1}$ model - applies to the last two regimes: $\Lambda \ll|\Delta m| \ll \xi^{1 / 2}$ (the left lower corner) and $\Delta m \rightarrow 0$ (the right lower corner). The latter case corresponds to the vanishing twisted mass.

is much weaker. Thus, we deal here with the formation of "almost" 't Hooft-Polyakov monopoles, with a typical size $\sim|\Delta m|^{-1}$. Only at much larger distances, $\sim \xi^{-1 / 2}$, the charge condensation enters the game, and forces the magnetic flux, rather than spreading evenly a lá Coulomb, to form flux tubes (the upper right corner of Fig. 1). There will be two such flux tubes, with the distinct orientation of the color-magnetic flux. The monopoles, albeit confined, are weakly confined.

Now, if we further reduce $|\Delta m|$,

$$
\Lambda \ll|\Delta m| \ll \sqrt{\xi},
$$

the size of the monopole $\left(\sim|\Delta m|^{-1}\right)$ becomes larger than the transverse size of the attached strings. The monopole gets squeezed in earnest by the strings - it becomes a bona fide confined monopole (the lower left corner of Fig. 1). A macroscopic description of such monopoles is provided by the twisted-mass $C P^{1}$ model, on which we will dwell below. The value of the twisted mass $\mu=\Delta m$. The confined monopole is nothing but the twisted-mass sigma-model kink which has a typical size $\sim|\mu|^{-1}$.

As we further diminish $|\Delta m|$ approaching $\Lambda$ and then getting below $\Lambda$, the size of the monopole grows, and, classically, it would explode. This is where quantum 
effects in the world-sheet theory take over. It is natural to refer to this domain of parameters as the "regime of highly quantum dynamics." While the thickness of the string (in the transverse direction) is $\sim \xi^{-1 / 2}$, the $z$-direction size of the kink representing the confined monopole in the highly quantum regime is much larger, $\sim \Lambda^{-1}$, see the lower right corner of Fig. 1 .

While the monopoles on the Coulomb branch (i.e. those of the 't Hooft-Polyakov type) are thoroughly discussed in the literature, the Higgs-branch monopoles - the confined monopoles - received much less attention. We intend to close this gap. We will study what becomes of the non-Abelian SU(2) monopole in the confinement phase which is set by the quark condensation and formation of the flux tubes (at nonvanishing FI parameter $\xi \neq 0$ ). The monopole-antimonopole pair will be confined in the meson-like state by a composite string, a bound state of two "elementary" strings [16], see Fig. 2a. Unfortunately, such mesons are unstable and cannot be studied in the static limit. Instead, we will focus on another (static) field configuration typical of the confinement phase: an $\mathrm{SU}(2)$ monopole with two semi-infinite elementary strings attached to it, see Fig. 2b. This configuration, a junction of two elementary strings, is stable and, moreover, 1/4-BPS saturated.

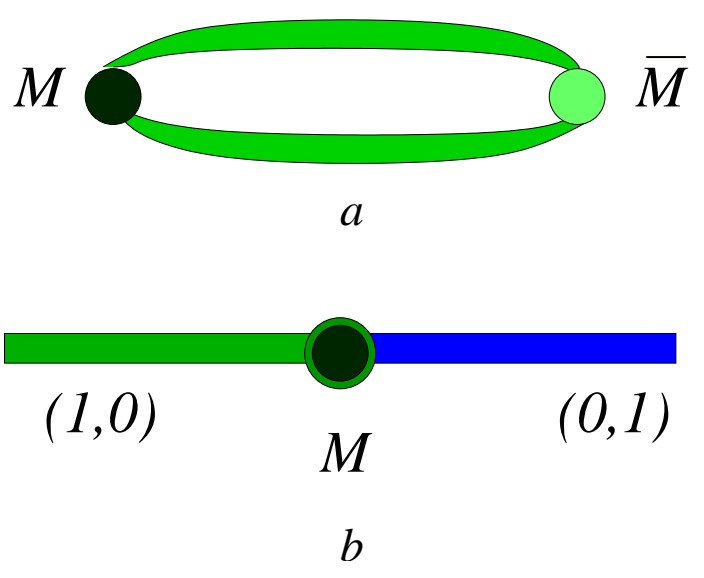

Figure 2: (a) The monopole-antimonopole pair; (b) the monopole with two (infinitely long) elementary flux tubes attached to it.

We perform a complete quasiclassical analysis of our microscopic model. A full set of the first-order master equations describing all $1 / 2$ and $1 / 4$ BPS topological defects - domain walls, strings, and all possible junctions - is derived. While the domain walls, strings and the wall-string junctions were studied previously, we expand the analysis to cover the case of the string junctions. We find appropriate solutions of the master equations. We derive zero modes, both bosonic and fermionic, specific for non-Abelian strings and their junctions. The corresponding collective coordinates (moduli) are introduced, which allows us to obtain the macroscopic description of the topological defects in question. We present solid quantitative evidence that at 
$\sqrt{\xi} \gg|\Delta m|$ the effective $(1+1)$-dimensional theory on the string world sheet is the $C P^{1}$ model, and that the confined monopoles of the microscopic theory must be identified with the kinks of the macroscopic $C P^{1}$ model. In particular, we explicitly demonstrate that the first-order BPS equations of the 4D Yang-Mills theory reduce to first-order BPS equations for the $C P^{1}$-model kink. We match the mass of the confined 4D Yang-Mills monopole with that of the $C P^{1}$-model kink. This evidence completes the proof of the statements that were made in the literature previously $[6,2]$.

The mass match mentioned above requires the presence of an anomaly in the monopole central charge of 4D Yang-Mills theory which must match its counterpart in the $C P^{1}$ model. While the anomaly in the $C P^{1}$ model was known previously [20], that in the Yang-Mills theory was not known. A fermion operator, an anomaly in the anticommutator $\{Q, Q\}$, crucial for the confined monopoles, was identified in our recent publication [2]. Here we further elaborate on this issue. Meanwhile, another anomalous contribution, crucial for the monopoles in the Coulomb regime, was identified and analyzed in Ref. [21].

The identification of the confined monopoles of 4D Yang-Mills theory with the kinks of the 2D $C P^{1}$ model gives us two advantages. First, we can and do explore the highly quantum regime of $\Delta m, \mu \rightarrow 0$. In this limit no quasiclassical treatment is available. The global $\mathrm{SU}(2)$ gets restored. Confined monopoles do not disappear, they survive. Appropriate exploration tools are available in the framework of the $C P^{1}$ model.

Second, we can and do explain a long-standing puzzling observation made in Ref. [4]. A comparison of the corresponding central charges revealed [4] a close parallel between four-dimensional Yang-Mills theory with $N_{f}=2$ and the twodimensional $C P^{1}$ model. The observation referred to the Coulomb branch of the Seiberg-Witten theory, with unconfined 't Hooft-Polyakov-like monopoles/dyons. We clarify physics responsible for this correspondence. In fact, the twisted-mass $C P^{1}$ model is equivalent to the Higgs phase of four-dimensional Yang-Mills theory with $N_{f}=2$. These two theories are "microscopic-macroscopic" partners. We show, however, that the BPS data are independent of the value of the FI parameter $\xi$, because this parameter has the $R$ parity that does not match that of the central charges. Therefore, in the BPS sector one can vary the FI parameter at will; in particular, pass to the limit $\xi \rightarrow 0$, where one finds oneself on the Coulomb branch. Thus, the bona fide correspondence refers to the Higgs phase where it is rather obvious, but holomorphy allows one to extend it to the Coulomb branch too.

The paper is organized as follows. In Sect. 2 we outline our microscopic 4D Yang-Mills theory: $\mathrm{SU}(2) \times \mathrm{U}(1)$ two-flavor $\mathrm{QCD}$ with the Fayet-Iliopoulos term and extended $\mathcal{N}=2$ supersymmetry. Section 3 is devoted to description of the non-Abelian strings and introduction of orientational moduli. In Sect. 4 we derive and study the effective 2D theory on the string world sheet. In particular, we show, 
by virtue of an explicit calculation, that a non-vanishing $\Delta m$ induces the twisted mass term $\mu$ in the world-sheet $C P^{1}$ model, and $\Delta m=\mu$. Next we calculate superorientational (fermion) zero modes of the non-Abelian string. In Sect. 5 we establish and exploit the correspondence between the monopole and the junction of two elementary strings. We solve the first-order master equations for the junction, match the monopole and kink masses, discuss the multiplicity matching and other implications for the confined monopoles. In Sect. 6 we consider monopoles/kinks in the quantum limit $\Delta m, \mu \rightarrow 0$ and match anomalies in the central charges of the corresponding superalgebras. In Sect. 7 we explain why the BPS sector of twodimensional $C P^{1}$ model is related to the Seiberg-Witten solution of 4D super-YangMills theory: a direct correspondence is between $C P^{1}$ and the Higgs phase of YangMills; holomorphy of the central charges makes possible a subsequent transition to the Coulomb branch (the Seiberg-Witten solution). Finally, Section 8 briefly summarizes our conclusions.

\section{A brief summary of the theoretical set-up}

Our task is to study the string/D-brane phenomena in supersymmetric gauge theories in a fully controllable mode. An appropriate theoretical set-up gradually emerged in the last three years $[16,1,3,2]$ - a particular $\mathcal{N}=2$ gauge model with judiciously chosen matter hypermultiplets and a special adjustment of the matter mass terms. The model evolved in the direction of simplification; currently it presents a theoretical scene fully fit for studies of the phenomena we are interested in.

The gauge symmetry of the model we will deal with is $\mathrm{SU}(2) \times \mathrm{U}(1)$. Besides the gauge bosons, gauginos and their $\mathcal{N}=2$ superpartners, it has a matter sector consisting of two "quark" hypermultiplets, with large (and degenerate or nearly degenerate) mass terms. One also introduces a Fayet-Iliopoulos term, so that the overall superpotential takes the form

$$
\begin{aligned}
\mathcal{W} & =\frac{1}{\sqrt{2}} \sum_{A=1}^{2}\left(\tilde{q}_{A} \mathcal{A} q^{A}+\tilde{q}_{A} \mathcal{A}^{a} \tau^{a} q^{A}\right) \\
& +\sum_{A=1,2} m_{A} q^{A} \tilde{q}_{A}-\frac{1}{\sqrt{2}} \xi \mathcal{A},
\end{aligned}
$$

where $\mathcal{A}^{a}$ and $\mathcal{A}$ are chiral superfields, the $\mathcal{N}=2$ superpartners of the gauge bosons of $\mathrm{SU}(2)$ and $\mathrm{U}(1)$, respectively. Furthermore, $q_{A}$ and $\tilde{q}_{A}(A=1,2)$ represent two matter hypermultiplets, while $\xi, m_{1}$ and $m_{2}$ are constants, assumed to be much larger than the dynamical scale parameter of the $\mathrm{SU}(2)$ gauge theory, see Sect. 3. The mass terms $m_{1,2}$ can always be made real and positive by virtue of an appropriate field definition. We will assume this to be the case. Moreover, for simplicity we 
will assume that the Fayet-Iliopoulos parameter $\xi$ is real and positive too. ${ }^{3}$ For further details of our theoretical set-up the reader is referred to Refs. [16, 3, 2]. Besides local symmetries, (and besides a global $\mathrm{SU}(2)_{R}$ inherent to $\mathcal{N}=2$ ), at $m_{1}=m_{2}$ the model has a global SU(2) flavor symmetry associated with rotations of the first and second hypermultiplets. This "symmetric" point is the focus of the present work. However, to make contact with quasiclassical results, at times we will leave the symmetric point $m_{1}=m_{2}$ and consider a deformed case $m_{1} \neq m_{2}$ assuming, however, the deformation to be small, $\left|m_{1}-m_{2}\right| \ll m_{1,2}$. The reader is advised to exercise caution not to confuse these two regimes.

The Fayet-Iliopoulos term triggers the spontaneous breaking of the gauge symmetry. The vacuum expectation values (VEV's) of the squark fields can be chosen as

$$
\begin{gathered}
\left\langle q^{k A}\right\rangle=\left\langle\overline{\tilde{q}}^{k A}\right\rangle=\sqrt{\frac{\xi}{2}}\left(\begin{array}{ll}
1 & 0 \\
0 & 1
\end{array}\right), \\
k=1,2, \quad A=1,2,
\end{gathered}
$$

up to gauge rotations. The color-flavor locked form of VEV's in Eq. (2.2) results in the fact that, while the theory is fully Higgsed, a diagonal $\mathrm{SU}(2)_{C+F}$ survives as a global symmetry (in the limit $m_{1}=m_{2}$ ). This is a particular case of the BardakciHalpern mechanism [24]. The most economic way to see the occurrence of the above global symmetry is through the matrix notation

$$
Q=\left(\begin{array}{ll}
q^{11} & q^{12} \\
q^{21} & q^{22}
\end{array}\right)
$$

(and the same for $\tilde{q}$ ). Here $Q$ is a $2 \times 2$ matrix, the first superscript refers to $\mathrm{SU}(2)$ "color" (we will also use the notation $\varphi^{r}$ and $\varphi^{b}$ meaning red and blue), while the second ( $A=1$ or 2 ) to "flavor." The covariant derivatives are defined in such a way that they act from the left,

$$
\nabla_{\mu} Q \equiv\left(\partial_{\mu}-\frac{i}{2} A_{\mu}-i A_{\mu}^{a} \frac{\tau^{a}}{2}\right) Q
$$

while the global flavor $\mathrm{SU}(2)$ transformations then act on $Q$ from the right. Equation (2.4) also shows our $\mathrm{U}(1)$ charge convention. Needless to say, $\mathcal{N}=2$ supersymmetry of the model is unaffected by the gauge symmetry breaking (2.2). The local and global symmetries of the model and of the vacuum state are summarized in Table 1. In the vacuum, there are no massless modes, all excitations are massive.

\footnotetext{
${ }^{3}$ In fact, $\xi$ is the first component of an $\mathrm{SU}(2)_{R}$ vector $\vec{\xi}$ of the generalized Fayet-Iliopoulos parameters introduced in $[22,23]$, see Eq. (3.35) below.
} 


\begin{tabular}{|c|c|}
\hline $\mathcal{N}=2$ SUSY & unbroken \\
\hline $\mathrm{SU}(2)_{R}$ & unbroken \\
\hline$\Delta m=0:\{\mathrm{U}(1) \times \mathrm{SU}(2)\}_{G} \times \mathrm{SU}(2) \times \mathrm{U}(1)$ & $\mathrm{U}(1)_{\operatorname{diag}} \times \mathrm{SU}(2)_{\operatorname{diag}}$ \\
\hline$\Delta m \neq 0:\{\mathrm{U}(1) \times \mathrm{SU}(2)\}_{G} \times \mathrm{U}(1) \times \mathrm{U}(1)$ & $\mathrm{U}(1)_{\operatorname{diag}}$ \\
\hline
\end{tabular}

Table 1: Symmetries of the microscopic theory and the pattern of the symmetry breaking in the vacuum.

Concluding this section it would be in order to present a broader perspective on our theoretical set-up. This will hopefully provide additional conceptual insights albeit technically this aspect will not be pursued. We can view our model as a descendant of an $\mathcal{N}=2$ theory with the $\mathrm{SU}(3)$ gauge group broken down to $\mathrm{SU}(2) \times \mathrm{U}(1)$ at a high scale, of the order of the mass parameters $m_{1,2}$. This $\mathrm{SU}(3)$ "proto" theory has three types of monopoles associated with three roots of the SU(3) algebra. Two of them are confined by "elementary" strings which we denote as $(1,0)$ and $(0,1)[16]$. (In general, $(n, k)$ denotes the string with two winding numbers $n$ and $k$ with respect to two $\mathrm{U}(1)$ Cartan subgroups of $\mathrm{SU}(3)$, see Sect. 3 for details.) These monopoles are very heavy - their masses are of the order of $\left(m_{1,2}\right) / g^{2}$ and we do not discuss them in this paper. Note, however, that they were considered recently in Ref. [17]. Our primary interest is the "third" monopole which lies entirely inside the $\mathrm{SU}(2)$ factor of the $\mathrm{SU}(3)$ gauge group. It is much lighter than the two mentioned above. This is the reason why in our studies we settle for the $\mathrm{SU}(2) \times \mathrm{U}(1)$ model while the full $\mathrm{SU}(3)$ model is behind the scene.

\section{Non-Abelian strings}

In this section we review the formalism of Ref. [3] where non-Abelian strings were first introduced, and make adjustments necessary for the present work. We start from $\mathrm{U}(1) \times \mathrm{U}(1)$ moduli-free string solutions found in Ref. [16] in the case $m_{1} \neq m_{2}$. Then we show how additional orientational zero modes arise in the limit $m_{1} \rightarrow m_{2}$ making the strings at hand non-Abelian [3].

Let us start from the case $m_{1} \neq m_{2}$. One can readily convince oneself that as far as the flux-tube solutions are concerned it is sufficient to limit oneself to the following ansatz for the matter fields:

$$
q^{k A}=\overline{\tilde{q}}^{k A} \equiv \frac{1}{\sqrt{2}} \varphi^{k A}
$$


Correspondingly, we introduce the matrix

$$
\Phi=\left(\begin{array}{ll}
\varphi^{11} & \varphi^{12} \\
\varphi^{21} & \varphi^{22}
\end{array}\right), \quad \Phi=\sqrt{2} Q=\sqrt{2} \overline{\tilde{Q}}
$$

where the first superscript refers to SU(2) color, while the second to flavor. Note that the field identification (3.1) is inappropriate in dealing with quantum corrections, and, in particular, in the zero-mode analysis.

Then the bosonic part of the effective action of the model at hand takes the form $^{4}$

$$
\begin{aligned}
S & =\int \mathrm{d}^{4} x\left\{\frac{1}{4 g_{2}^{2}}\left(F_{\mu \nu}^{a}\right)^{2}+\frac{1}{4 g_{1}^{2}}\left(F_{\mu \nu}\right)^{2}+\frac{1}{g_{1}^{2}}\left|\partial_{\mu} a\right|^{2}+\frac{1}{g_{2}^{2}}\left|D_{\mu} a^{a}\right|^{2}\right. \\
& +\operatorname{Tr}\left(\nabla_{\mu} \Phi\right)^{\dagger}\left(\nabla_{\mu} \Phi\right)+\frac{g_{2}^{2}}{8}\left[\operatorname{Tr}\left(\Phi^{\dagger} \tau^{a} \Phi\right)\right]^{2}+\frac{g_{1}^{2}}{8}\left[\operatorname{Tr}\left(\Phi^{\dagger} \Phi\right)-2 \xi\right]^{2} \\
& \left.+\frac{1}{2} \operatorname{Tr}\left\{\left(\Phi^{\dagger}\left[a_{a} \tau^{a}+a\right]^{\dagger}+\sqrt{2} M^{\dagger} \Phi^{\dagger}\right)\left(\left[a_{a} \tau^{a}+a\right] \Phi+\Phi \sqrt{2} M\right)\right\}\right\},
\end{aligned}
$$

where $a$ is the lowest component of the chiral superfield $\mathcal{A}$. Here we introduced a $2 \times 2$ mass matrix $M$ acting on the flavor indices of $\Phi$. With our convention $M$ is diagonal,

$$
M=\left(\begin{array}{cc}
m_{1} & 0 \\
0 & m_{2}
\end{array}\right)
$$

Equation (3.3) implies, that besides the squark VEV's (2.2), the field $a$ and $a^{a}$ develop vacuum expectations value too,

$$
a=-\sqrt{2} m, \quad a^{3}=-\frac{1}{\sqrt{2}} \Delta m,
$$

where

$$
m=\frac{1}{2}\left(m_{1}+m_{2}\right), \quad \Delta m=m_{1}-m_{2} .
$$

We see that at $m_{1} \neq m_{2}$ the $\mathrm{SU}(2) \times \mathrm{U}(1)$ gauge symmetry is broken down to $\mathrm{U}(1) \times \mathrm{U}(1)$ by the VEV of the adjoint scalar field $a^{3}$. For definiteness, we will

\footnotetext{
${ }^{4}$ Here and below we use a formally Euclidean notation, e.g. $F_{\mu \nu}^{2}=2 F_{0 i}^{2}+F_{i j}^{2},\left(\partial_{\mu} a\right)^{2}=$ $\left(\partial_{0} a\right)^{2}+\left(\partial_{i} a\right)^{2}$, etc. This is appropriate since we are going to study static (time-independent) field configurations, and $A_{0}=0$. Then the Euclidean action is nothing but the energy functional. Furthermore, we define $\sigma^{\alpha \dot{\alpha}}=(1,-i \vec{\tau}), \bar{\sigma}_{\dot{\alpha} \alpha}=(1, i \vec{\tau})$. Lowing and raising of spinor indices is performed by virtue of the antisymmetric tensor defined as $\varepsilon_{12}=\varepsilon_{\dot{1} \dot{2}}=1, \varepsilon^{12}=\varepsilon^{i \dot{2}}=-1$. The same raising and lowering convention applies to the flavor $\mathrm{SU}(2)$ indices $f, g$, etc.
} 
assume that $m_{1}>m_{2}$. Then $\Delta m$ is positive. ${ }^{5}$ If $|\Delta m| \gg \xi^{1 / 2}$ then the gauge symmetry breaking by $\left\langle a^{3}\right\rangle$ has a larger scale than that by the squark fields. This is a more important effect leading to monopoles with masses $\sim|\Delta m| / g_{2}^{2}$. Formation of strings is governed by $\xi$ and can be viewed as a "secondary" effect.

On the other hand, if $\Delta m=0$, the gauge $\mathrm{SU}(2)$ group is unbroken by the adjoint scalar VEV's, since they reduce to

$$
a=-\sqrt{2} m, \quad a^{3}=0 .
$$

With two matter hypermultiplets, the $\mathrm{SU}(2)$ part of the gauge group is asymptotically free, implying generation of a dynamical scale $\Lambda$. If descent to $\Lambda$ were uninterrupted, the gauge coupling $g_{2}^{2}$ would explode at this scale. Moreover, strong coupling effects in the $\mathrm{SU}(2)$ subsector at the scale $\Lambda$ would break the $\mathrm{SU}(2)$ subgroup through the Seiberg-Witten mechanism [11]. Since we want to stay at weak coupling we assume that $\sqrt{\xi} \gg \Lambda$, so that the $\mathrm{SU}(2)$ coupling running is frozen by the squark condensation at a small value

$$
\frac{8 \pi^{2}}{g_{2}^{2}}=2 \ln \frac{\sqrt{\xi}}{\Lambda}+\cdots \gg 1
$$

Alternatively one can say that

$$
\Lambda^{2}=\xi \exp \left(-\frac{8 \pi^{2}}{g_{2}^{2}(\xi)}\right)
$$

with $g_{2}^{2}(\xi) \ll 1$.

\subsection{Abrikosov-Nielsen-Olesen vs. elementary strings}

To warm up, let us discuss the conventional Abrikosov-Nielsen-Olesen (ANO) string [25] in our model. ${ }^{6}$ The existence of the ANO string is due to the fact that $\pi_{1}(\mathrm{U}(1))=Z$, ensuring its topological stability. For this solution one can discard the $\mathrm{SU}(2)_{c}$ part of the action ( $c$ stands for "color"), putting $A_{\mu}^{a}=a^{a} \equiv 0$. Correspondingly, there is no $\mathrm{SU}(2)$ winding of $\Phi$. Non-trivial topology is realized through the $\mathrm{U}(1)$ winding of $\Phi$,

$$
\Phi(x)=\sqrt{\xi} e^{i \alpha(x)}, \quad|x| \rightarrow \infty,
$$

\footnotetext{
${ }^{5}$ To keep our notation concise we will also use the parameter $\mu \equiv m_{1}-m_{2} \equiv \Delta m$. This parameter is not to be confused with the $\mathcal{N}=2$ breaking perturbation, e.g. Refs. [1, 2], which is routinely denoted by $\mu$ following Seiberg and Witten [11, 12]. In most cases $\mu$ will be reserved for the twisted mass in the macroscopic $C P^{1}$ model, while the mass splitting $\Delta m$ will be used in the microscopic 4D Yang-Mills theory. We will show that $\mu=\Delta m$. In some sections (e.g. Sects. 4.3 and 6), for brevity, $\mu$ will replace $\Delta m$ in the microscopic 4D Yang-Mills theory.

${ }^{6}$ This subsection is insensitive with regards to the choice of $\Delta m$ which may or may not vanish.
} 
and

$$
A_{\ell}=-2 \varepsilon_{\ell k} \frac{x_{k}}{r}, \quad \ell, k=1,2,
$$

where $\alpha$ is the angle in the perpendicular plane (Fig. 3), and $r$ is the distance from the string axis in the perpendicular plane. Equations (3.10) and (3.11) refer to the minimal ANO string, with the minimal winding. Needless to say, the tension of the

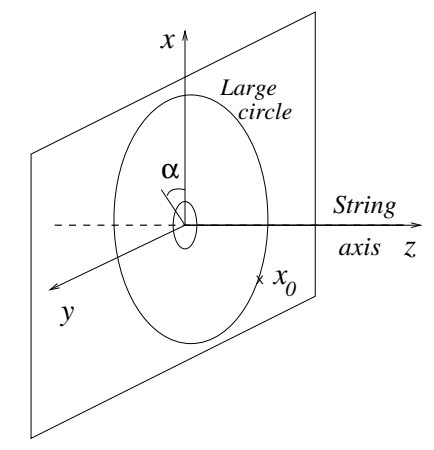

Figure 3: Geometry of the string.

ANO string is given by the standard formula

$$
T_{\mathrm{ANO}}=4 \pi \xi .
$$

This is not the string we are interested in here, however - in fact, in the problem at hand there are "more elementary" strings with $1 / 2$ of the above tension, so that the ANO string can be viewed as a bound state of two elementary strings.

Where do they come from? Since $\pi_{1}(\mathrm{SU}(2))$ is trivial, at first sight it might seem that there are no new options. This conclusion is wrong - one can combine the $Z_{2}$ center of $\mathrm{SU}(2)$ with the element $-1 \in \mathrm{U}(1)$ to get a topologically stable string-like solution possessing both windings, in $\mathrm{SU}(2)$ and $\mathrm{U}(1)$, of the following type

$$
\begin{aligned}
\Phi(x) & =\sqrt{\xi} \exp \left[i \alpha(x) \frac{1 \pm \tau^{3}}{2}\right], \quad|x| \rightarrow \infty, \\
A_{\ell} & =-\varepsilon_{\ell k} \frac{x_{k}}{r}, \quad A_{\ell}^{3}=\mp \varepsilon_{\ell k} \frac{x_{k}}{r}, \quad \ell, k=1,2,
\end{aligned}
$$

Correspondingly, the U(1) magnetic flux is twice smaller than in the ANO case. Since it is only the U(1) magnetic flux that enters the expression for the appropriate central charge (see below), the tension of the flux tube generated by the winding $(3.13)$ is

$$
T_{ \pm}=2 \pi \xi
$$

The \pm subscript corresponds to two types of elementary strings in which either only $\varphi^{r}$ or only $\varphi^{b}$ are topologically non-trivial. 
We will refer to the strings corresponding to the boundary conditions (3.13) as $(1,0)$ and $(0,1)$ for the following reasons. For the case of non-equal quark masses the $\mathrm{SU}(2) \times \mathrm{U}(1)$ group is broken by the adjoint scalar $\mathrm{VEV}$ to $\mathrm{U}(1) \times \mathrm{U}(1)$. We have a lattice of strings labeled by two integer numbers $(n, k)$ associated with the windings with respect to two gauge $\mathrm{U}(1)$ groups which are linear combinations of the two $\mathrm{U}(1)$ 's above which are natural in the $\mathrm{SU}(3)$ "proto" theory. In this terminology the ANO string is the sum $(0,1)+(1,0)=(1,1)$, see [16] for further details.

\subsection{Embedding; first-order equations for the elementary strings}

The charges of the $(n, k)$-strings can be plotted in the Cartan plane of the $\mathrm{SU}(3)$ algebra of the "proto" theory. We will use the convention of labeling the flux of a given string by the magnetic charge of the monopole which produces this flux and can be attached to its end. This is possible since both the string fluxes and the monopole charges are elements of the group $\pi_{1}\left(U(1)^{2}\right)=Z^{2}$. This convention is convenient because specifying the flux of a given string automatically fixes the charge of the monopole that it confines.

Our strings are formed by the condensation of squarks which have electric charges equal to the weights of the $\mathrm{SU}(3)$ algebra. The Dirac quantization condition tells us [16] that the lattice of the $(n, k)$-strings is formed by the roots of the $\mathrm{SU}(3)$ algebra. This lattice of the $(n, k)$-strings is shown in Fig. 4 . Two strings $(1,0)$ and $(0,1)$ are the "elementary" or "minimal" BPS strings. All other strings can be considered as bound states of these elementary strings. If we plot two lines along the charges of these elementary strings (see Fig. 4) they divide the lattice into four sectors. It turns out [16] that the strings in the upper and lower sectors are BPS but they are marginally unstable. On the contrary, the strings lying in the right and left sectors are (meta)stable bound states of the elementary ones; they are not BPS-saturated.

Our study objective is the elementary string. Since the both strings, $(0,1)$ and $(1,0)$, are 1/2 BPS-saturated, Eq. (3.14) is exact. These strings are absolutely degenerate. The degeneracy holds beyond the classical level, with inclusion of quantum corrections, perturbative and nonperturbative. This is important for what follows. The existence of two distinct strings with exactly degenerate tensions is a special feature of supersymmetry implying the existence of a 2-string junction. A similar phenomenon for domain walls - 2-wall junctions — was studied recently [26].

The first-order equations for the BPS strings following from the action (3.3) are

$$
\begin{aligned}
& F_{3}^{* a}+\frac{g_{2}^{2}}{2} \sigma\left(\bar{\varphi}_{A} \tau^{a} \varphi^{A}\right)=0, \quad a=1,2,3 \\
& F_{3}^{*}+\frac{g_{1}^{2}}{2} \sigma\left(\left|\varphi^{A}\right|^{2}-2 \xi\right)=0 ;
\end{aligned}
$$




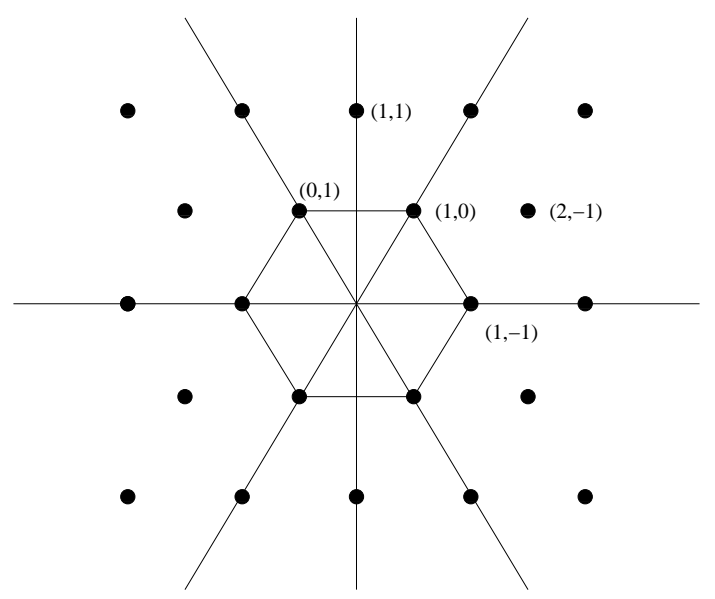

Figure 4: Lattice of $(n, k)$ vortices.

$$
\left(\nabla_{1}+i \sigma \nabla_{2}\right) \varphi^{A}=0,
$$

where $\sigma= \pm 1$ is the sign of the total flux and

$$
F_{m}^{*}=\frac{1}{2} \varepsilon_{m n k} F_{n k}, \quad m, n, k=1,2,3 .
$$

(see Ref. [3] and Sect. 5.1). To construct the $(0,1)$ and $(1,0)$ strings we further restrict the gauge field $A_{\mu}^{a}$ to the single color component $A_{\mu}^{3}$, setting $A_{\mu}^{1}=A_{\mu}^{2}=0$, and consider only the squark fields of the $2 \times 2$ color-flavor diagonal form,

$$
\varphi^{k A}(x) \neq 0, \quad \text { for } \quad k=A=1,2 .
$$

The off-diagonal components of the matrix $\Phi$ are set to zero.

The $(1,0)$ string arises when the first flavor has the unit winding number while the second flavor does not wind at all. And vice versa, the $(0,1)$ string arises when the second flavor has the unit winding number while the first flavor does not wind. Consider for definiteness the $(1,0)$ string. The solutions of the first-order equations (3.15) will be sought for using the following ansatz [16]:

$$
\begin{aligned}
\Phi(x) & =\left(\begin{array}{cc}
e^{i \alpha} \phi_{1}(r) & 0 \\
0 & \phi_{2}(r)
\end{array}\right), \\
A_{i}^{3}(x) & =-\varepsilon_{i j} \frac{x_{j}}{r^{2}}\left(1-f_{3}(r)\right), \\
A_{i}(x) & =-\varepsilon_{i j} \frac{x_{j}}{r^{2}}(1-f(r))
\end{aligned}
$$


where the profile functions $\phi_{1}, \phi_{2}$ for the scalar fields and $f_{3}, f$ for the gauge fields depend only on $r(i, j=1,2)$. Applying this ansatz one can rearrange [16] the first-order equations (3.15) in the form

$$
\begin{aligned}
& r \frac{d}{d r} \phi_{1}(r)-\frac{1}{2}\left(f(r)+f_{3}(r)\right) \phi_{1}(r)=0, \\
& r \frac{d}{d r} \phi_{2}(r)-\frac{1}{2}\left(f(r)-f_{3}(r)\right) \phi_{2}(r)=0, \\
& -\frac{1}{r} \frac{d}{d r} f(r)+\frac{g_{1}^{2}}{2}\left[\left(\phi_{1}(r)\right)^{2}+\left(\phi_{2}(r)\right)^{2}-2 \xi\right]=0, \\
& -\frac{1}{r} \frac{d}{d r} f_{3}(r)+\frac{g_{2}^{2}}{2}\left[\left(\phi_{1}(r)\right)^{2}-\left(\phi_{2}(r)\right)^{2}\right]=0 .
\end{aligned}
$$

Furthermore, one needs to specify the boundary conditions which would determine the profile functions in these equations. Namely,

$$
\begin{aligned}
& f_{3}(0)=1, \quad f(0)=1 ; \\
& f_{3}(\infty)=0, \quad f(\infty)=0
\end{aligned}
$$

for the gauge fields, while the boundary conditions for the squark fields are

$$
\phi_{1}(\infty)=\sqrt{\xi}, \quad \phi_{2}(\infty)=\sqrt{\xi}, \quad \phi_{1}(0)=0 .
$$

Note that since the field $\phi_{2}$ does not wind, it need not vanish at the origin, and it does not. Numerical solutions of the Bogomolny equations (3.19) for the $(0,1)$ and $(1,0)$ strings were found in Ref. [3], see e.g. Figs. 1 and 2 in this paper.

\subsection{Non-Abelian moduli}

Now let us assume $\Delta m=0$ and demonstrate the occurrence of a more general solution [3] which contains non-Abelian moduli. The adjoint scalar VEV does not break the gauge $\mathrm{SU}(2)$ if $\Delta m=0$. The relevant homotopy group in this case is the fundamental group

$$
\pi_{1}\left(\frac{\mathrm{SU}(2) \times \mathrm{U}(1)}{Z_{2}}\right)=Z .
$$

This means that the $(n, k)$-string lattice reduces to a tower labeled by a single integer $(n+k)$. For instance, the $(1,-1)$ string becomes classically unstable (no barrier). On the SU(2) group manifold it corresponds to a winding along the equator on the sphere $S_{3}$ (Fig. 5). Clearly this winding can be shrunk to zero by contracting the 


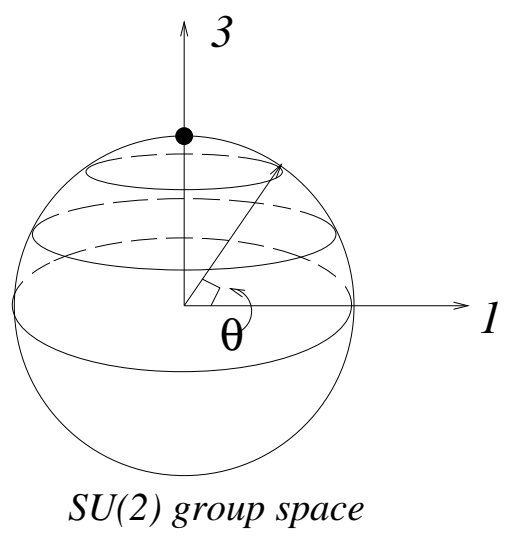

Figure 5: Unwinding the (1,-1)-string.

loop towards north or south poles [27]. On the other hand, the elementary $(1,0)$ and $(0,1)$ strings cannot be shrunk, as was explained above. They correspond to a half-circle winding along the equator. The $(1,0)$ and $(0,1)$ strings form a doublet of the residual global $\mathrm{SU}(2)_{C+F}$.

A remarkable feature of the $(1,0)$ and $(0,1)$ strings is the occurrence of nonAbelian moduli which are absent in the ANO strings. Indeed, while the vacuum field $\Phi_{\mathrm{vac}}=\sqrt{\xi} I$ (here $I$ is $2 \times 2$ unit matrix) is invariant under the global $\mathrm{SU}(2)_{C+F}$,

$$
\Phi \rightarrow U_{L} \Phi U_{R}, \quad U_{R}=U_{L}^{\dagger},
$$

the string configuration (3.18) is not. ${ }^{7}$ Therefore, if there is a solution of the form (3.18), there is in fact a family of solutions obtained from (3.18) by the combined global gauge-flavor rotation. Say, for quark fields it reads

$$
\Phi(x) \rightarrow e^{i \beta} e^{i \vec{\omega} \vec{\tau} / 2} \Phi(x) e^{-i \beta} e^{-i \vec{\omega} \vec{\tau} / 2} .
$$

The $\mathrm{U}(1)$ factor $e^{i \beta}$ does not act on the string solution (3.18). It is not to be counted. Thus, what remains is $\mathrm{SU}(2)$. In fact, it is the coset $\mathrm{SU}(2) / \mathrm{U}(1)$, as is rather obvious from Eq. (3.18): rotations around the third axis in the $\mathrm{SU}(2)$ space leave the solution with the asymptotics (3.18) intact.

Thus, the introduction of the "moduli matrix" $U$ allows one to get a generic solution of the non-Abelian string Bogomolny equation with the following asymptotics at $|x| \rightarrow \infty$ :

$$
\Phi(x)=\sqrt{\xi} \exp \left(i \frac{\alpha(x)}{2}\right) \exp \left(i \alpha(x) \frac{\vec{n} \vec{\tau}}{2}\right),
$$

where $\vec{n}$ is a moduli vector defined as

$$
n^{a} \tau^{a}=U \tau^{3} U^{-1}, \quad a=1,2,3 .
$$

\footnotetext{
${ }^{7}$ Below we will drop the subscripts $R$ and $L$.
} 
It is subject to the condition

$$
\vec{n}^{2}=1 \text {. }
$$

At $n=\{0,0, \pm 1\}$ we get the field configurations quoted in Eq. (3.13). Every given matrix $U$ defines the moduli vector $\vec{n}$ unambiguously. The inverse is not true. If we consider the left-hand side of Eq. (3.26) as given, then the solution for $U$ is obviously ambiguous, since for any solution $U$ one can construct two "gauge orbits" of solutions, namely,

$$
\begin{aligned}
& U \rightarrow U \exp \left(i \alpha \tau_{3}\right), \\
& U \rightarrow \exp (i \beta \vec{n} \vec{\tau}) U .
\end{aligned}
$$

We will use this freedom in what follows.

At finite $|x|$ the non-Abelian string centered at the origin can be written as [3]

$$
\begin{aligned}
\Phi(x) & =U\left(\begin{array}{cc}
e^{i \alpha} \phi_{1}(r) & 0 \\
0 & \phi_{2}(r)
\end{array}\right) U^{-1} \\
& =e^{\frac{i}{2} \alpha\left(1+n^{a} \tau^{a}\right)} U\left(\begin{array}{cc}
\phi_{1}(r) & 0 \\
0 & \phi_{2}(r)
\end{array}\right) U^{-1}, \\
A_{i}^{a}(x) & =-n^{a} \varepsilon_{i j} \frac{x_{j}}{r^{2}}\left[1-f_{3}(r)\right], \\
A_{i}(x) & =-\varepsilon_{i j} \frac{x_{j}}{r^{2}}[1-f(r)],
\end{aligned}
$$

where the profile functions are the solutions to Eq. (3.19). Note that

$$
U\left(\begin{array}{cc}
\phi_{1} & 0 \\
0 & \phi_{2}
\end{array}\right) U^{-1}=\frac{\phi_{1}+\phi_{2}}{2}+n^{a} \tau^{a} \frac{\phi_{1}-\phi_{2}}{2} .
$$

Now it is particularly clear that this solution smoothly interpolates between the $(1,0)$ and $(0,1)$ strings: if $n=(0,0,1)$ the first-flavor $r$ squark winds at infinity while for $n=(0,0,-1)$ it is the second-flavor $b$ squark.

Since the $\mathrm{SU}(2)_{C+F}$ symmetry is not broken by the squark vacuum expectation values, it is physical and has nothing to do with the gauge rotations eaten by the Higgs mechanism. The orientational moduli $\vec{n}$ are not gauge artifacts. To see this we can construct gauge invariant operators which have explicit $\vec{n}$-dependence. Such a construction is convenient in order to elucidate features of our non-Abelian string solution as well as for other purposes. 


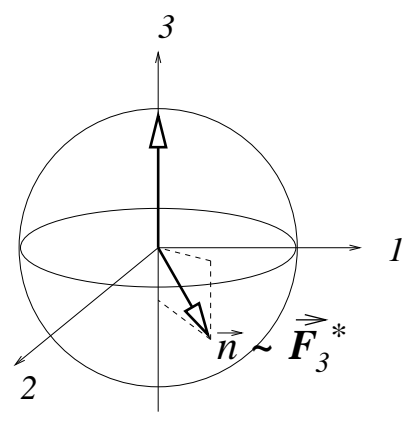

Figure 6: Bosonic moduli $n^{a}$ describe the orientation of the color-magnetic flux of the $(0,1)$ and $(1,0)$ strings in the $\mathrm{O}(3)$ group space, Eq. (3.32).

As an example, let us define the "non-Abelian" field strength (to be denoted by bold letters),

$$
\mathbf{F}_{3}^{* a}=\frac{1}{\xi} \operatorname{Tr}\left(\Phi^{\dagger} F_{3}^{* b} \frac{\tau^{b}}{2} \Phi \tau^{a}\right),
$$

where the subscript 3 marks the $z$ axis, the direction of the string (Fig. 3). From the very definition it is clear that this field is gauge invariant. ${ }^{8}$ Moreover, Eq. (3.29) implies that

$$
\mathbf{F}_{3}^{* a}=-n^{a} \frac{\left(\phi_{1}^{2}+\phi_{2}^{2}\right)}{2 \xi} \frac{1}{r} \frac{d f_{3}}{d r} .
$$

From this formula we readily infer the physical meaning of the moduli $\vec{n}$ : the flux of the color-magnetic field ${ }^{9}$ in the flux tube is directed along $\vec{n}$ (Fig. 6). For strings in Eq. (3.18), see also Eq. (3.13), the color-magnetic flux is directed along the third axis in the $\mathrm{O}(3)$ group space, either upward or downward. It is just this aspect that allows us to refer to the strings above as "non-Abelian." They are as non-Abelian as it gets at weak coupling.

Although the flux tubes in non-Abelian theories at weak coupling were studied in numerous papers in recent years $[28,29,30,31,32,33,16]$, in the previous constructions the flux was always directed in a fixed group direction (corresponding to a Cartan subalgebra), and no moduli that would freely govern its orientation in the group space were ever obtained.

To conclude this section let us present the non-Abelian string solution (3.29) in the singular gauge in which the squark fields at $r \rightarrow \infty$ tend to fixed VEV's and do not wind (i.e. do not depend on the polar angle $\alpha$ at $|x| \rightarrow \infty$ in the $x_{1,2}$ plane). In

\footnotetext{
${ }^{8}$ In the vacuum, where the matrix $\Phi$ is that of VEV's, $\mathbf{F}_{3}^{* a}$ and $F_{3}^{* a}$ would coincide.

${ }^{9}$ Defined in the gauge-invariant way, see Eq. (3.31).
} 
the singular gauge we have

$$
\begin{aligned}
\Phi & =U\left(\begin{array}{cc}
\phi_{1}(r) & 0 \\
0 & \phi_{2}(r)
\end{array}\right) U^{-1} \\
A_{i}^{a}(x) & =n^{a} \varepsilon_{i j} \frac{x_{j}}{r^{2}} f_{3}(r) \\
A_{i}(x) & =\varepsilon_{i j} \frac{x_{j}}{r^{2}} f(r) .
\end{aligned}
$$

In this gauge the spatial components of $A_{\mu}$ fall fast at large distances. If the chromomagnetic flux is defined as a circulation of $A_{i}$ over a circle encompassing the string axis, the flux will be saturated by the integral coming from the small circle around the (singular) string origin. We will use this singular-gauge form of the string solution later.

\subsection{Supersymmetry}

So far the $\mathcal{N}=2$ SUSY nature of the model at hand was behind the scene. Now it is in order to discuss this aspect. The central charge relevant to flux tubes is the so-called $(1 / 2,1 / 2)$ central charge [34]; in $\mathcal{N}=2$ theory it can be written as follows [23]:

$$
\left\{Q_{\alpha}^{f} \bar{Q}_{\dot{\beta} g}\right\}=2 \delta_{g}^{f}\left(\sigma_{\mu}\right)_{\alpha \dot{\beta}} P_{\mu}+4 i\left(\sigma_{\mu}\right)_{\alpha \dot{\beta}} \xi_{g}^{f} \int d^{3} x \frac{1}{2} \varepsilon_{0 \mu \delta \gamma} F_{\delta \gamma},
$$

where $P_{\mu}$ is energy-momentum operator, while ${ }^{10}$

$$
\xi_{g}^{f}=\left(\tau^{m} / 2\right)_{g}^{f} \xi^{m}
$$

where $\xi^{m}$ is an $\mathrm{SU}(2)_{R}$ triplet of the Fayet-Iliopoulos parameters. In the model under consideration only $\xi^{1} \equiv \xi$ is non-zero, so that

$$
\xi_{g}^{f}=\frac{\xi}{2}\left(\tau_{1}\right)_{g}^{f} .
$$

The central charge is the second term in Eq. (3.34). Note that it is only the U(1) field strength tensor that appears in this central charge. Moreover, it is obvious that

$$
\int d^{3} x \frac{1}{2} \varepsilon_{0 \mu \delta \gamma} F_{\delta \gamma}=L n_{\mu} \int d x d y F_{3}^{*}=L n_{\mu} \times(\text { Magnetic Flux })=2 \pi L n_{\mu},
$$

where $L$ is the string length and $n_{\mu}$ is the unit vector pointing in the direction of the string axis (which coincides with the $z$ axis in our convention, see Fig. 3.) The

\footnotetext{
${ }^{10}$ The factor $i$ in the second term on the right-hand side is due to our "Euclidean" notation, see footnote to Eq. (3.3). Note that the definition of $\xi_{g}^{f}$ in Ref. [23] differs by a factor of 2 .
} 
strings in question are 1/2 BPS-saturated so that (i) the combination of equations (3.34) and (3.37) implies that the tensions (3.14) are exact, and (ii) a "macroscopic" world-sheet theory must have four supercharges, i.e. must be $\mathcal{N}=2$ in terms of twodimensional world-sheet classification. This is because the original "microscopic" theory has eight supercharges. Note that the Fayet-Iliopoulos parameter is not renormalized by quantum corrections.

The flux tube is characterized by two translational moduli, $x_{0}$ and $y_{0}$, the positions of the string center in the perpendicular plane. The four supercharges that act non-trivially supplement $x_{0}$ and $y_{0}$ by four fermionic superpartners $\eta^{i}(i=1,2,3,4)$, supertranslational moduli. As usual, the (super)translational moduli split (i.e. completely decouple) from those describing internal dynamics. The dimension of representation is four, but this is a trivial multiplicity which we do not count when we speak of distinct elementary strings. In fact, a "distinct" string means that we deal with distinct dimension-four supermultiplets.

We will return to $\mathcal{N}=2$ superalgebra, in the anticommutator $\left\{Q_{\alpha}^{f} Q^{g}{ }_{\beta}\right\}$ relevant to the issue of string junctions (confined monopoles), in Sect. 6.

\section{Macroscopic theory}

In this section we will derive an effective low-energy theory for orientational collective coordinates on the string world sheet. As was already mentioned, this macroscopic theory is a two-dimensional supersymmetric $C P^{1}$ model. This model is discussed in great detail in the review paper [35] from which we borrow many definitions, notation and normalizations. At first we will review derivation [3] of the effective theory for the bosonic moduli $n^{a}$. In Sects. 4.1, 4.2 the mass difference is set to zero, $\Delta m=0$. Switching on $\Delta m$ will lead us to the $C P^{1}$ model with a "twisted mass." Then we will work out the fermionic part.

\subsection{Deriving the kinetic term; a basic normalizing integral}

Assume that the orientational collective coordinates $n^{a}$ are slow varying functions of the string world-sheet coordinates $x_{k}, k=0,3$. Then the moduli $n^{a}$ become fields of a (1+1)-dimensional sigma model on the world sheet. Since the vector $n^{a}$ parametrizes the string zero modes, there is no potential term in this sigma model. We begin with the kinetic term [3].

To obtain the kinetic term we substitute our solution, which depends on the moduli $\vec{n}$, in the action (3.3) assuming that the fields acquire a dependence on the coordinates $x_{k}$ via $n^{a}\left(x_{k}\right)$. Technically it is convenient to work with the solution (3.33) in the singular gauge. In doing so we immediately observe that we must modify the solution. 
Indeed, Eq. (3.30) or (3.33) were obtained as an $\mathrm{SU}(2)_{C+F}$ rotation of the "basic" $(1,0)$-string (3.18). Now we make this transformation local (i.e. depending on $x_{k}$ ). Because of this, the 0 and $z$ components of the gauge potential no longer vanish. They must be added to the ansatz. This situation is quite familiar (e.g. [27]) since one routinely encounters it in the soliton studies.

We suggest an obvious ansatz for these components (to be checked a posteriori),

$$
A_{k}=-i\left(\partial_{k} U\right) U^{-1} \rho(r), \quad k=0,3,
$$

where a new profile function $\rho(r)$ is introduced. To be more precise, we must express the right-hand side in terms of our moduli fields $n^{a}$. As was mentioned after Eq. (3.27), the parametrization of the matrix $U$ is ambiguous. Correspondingly, if we introduce

$$
\alpha_{k} \equiv-i\left(\partial_{k} U\right) U^{-1}, \quad \alpha_{k} \equiv \alpha_{k}^{a}\left(\frac{\tau^{a}}{2}\right)
$$

then $\alpha_{k}^{a}$ is defined modulo two gauge transformations following from Eq. (3.28). Equation (3.26) implies that

$$
\alpha_{k}^{a}-n^{a}\left(n^{b} \alpha_{k}^{b}\right)=-\varepsilon^{a b c} n^{b} \partial_{k} n^{c},
$$

and we can impose the condition $n^{b} \alpha_{k}^{b}=0$. Then

$$
\alpha_{k}^{a}=-\varepsilon^{a b c} n^{b} \partial_{k} n^{c}, \quad-i\left(\partial_{k} U\right) U^{-1}=-\frac{1}{2} \tau^{a} \varepsilon^{a b c} n^{b} \partial_{k} n^{c} .
$$

The function $\rho(r)$ in Eq. (4.1) is determined through a minimization procedure (cf. [27]) which generates $\rho$ 's own equation of motion. Our task is to derive it. But at first we note that $\rho(r)$ vanishes at infinity,

$$
\rho(\infty)=0
$$

The boundary condition at $r=0$ will be determined shortly.

The kinetic term for $n^{a}$ comes from the gauge and quark kinetic terms in Eq. (3.3). Using (3.33) and (4.1) to calculate the $\mathrm{SU}(2)$ gauge field strength we find

$$
F_{k i}=\frac{1}{2}\left(\partial_{k} n^{a}\right) \tau^{a} \varepsilon_{i j} \frac{x_{j}}{r^{2}} f_{3}[1-\rho(r)]+i\left(\partial_{k} U\right) U^{-1} \frac{x_{i}}{r} \frac{d \rho(r)}{d r} .
$$

We see that in order to have a finite contribution from $\operatorname{Tr} F_{k i}^{2}$ in the action we have to impose the constraint

$$
\rho(0)=1
$$

Substituting the field strength (4.6) in the action (3.3) and including, in addition, the kinetic term of quarks, we arrive at

$$
S^{(1+1)}=\frac{\beta}{2} \int d t d z\left(\partial_{k} n^{a}\right)^{2}
$$


where the coupling constant $\beta$ is given by a normalizing integral

$$
\begin{aligned}
\beta & =\frac{2 \pi}{g_{2}^{2}} \int_{0}^{\infty} r d r\left\{\left(\frac{d}{d r} \rho(r)\right)^{2}+\frac{1}{r^{2}} f_{3}^{2}(1-\rho)^{2}\right. \\
& \left.+g_{2}^{2}\left[\frac{\rho^{2}}{2}\left(\phi_{1}^{2}+\phi_{2}^{2}\right)+(1-\rho)\left(\phi_{1}-\phi_{2}\right)^{2}\right]\right\} .
\end{aligned}
$$

We will have to deal with the integral on the right-hand side more than once in what follows. There are various arguments allowing one to find it analytically; however, the first calculation was carried out numerically by R. Auzzi to whom we are deeply grateful.

The functional (4.9) must be minimized with respect to $\rho$ with the boundary conditions given by $(4.5),(4.7)$. Varying (4.9) with respect to $\rho$ one readily obtains the second-order equation which the function $\rho$ must satisfy,

$$
-\frac{d^{2}}{d r^{2}} \rho-\frac{1}{r} \frac{d}{d r} \rho-\frac{1}{r^{2}} f_{3}^{2}(1-\rho)+\frac{g_{2}^{2}}{2}\left(\phi_{1}^{2}+\phi_{2}^{2}\right) \rho-\frac{g_{2}^{2}}{2}\left(\phi_{1}-\phi_{2}\right)^{2}=0 .
$$

The second-order equation occurs because, once we allow the dependence of $n^{a}$ on the world-sheet coordinates $t, z$, the string is no longer BPS saturated. After some algebra and extensive use of the first-order equations (3.19) one can show that the solution of (4.10) is given by

$$
\rho=1-\frac{\phi_{1}}{\phi_{2}}
$$

This solution satisfies the boundary conditions (4.5) and (4.7), as it should.

Substituting this solution back into the expression for the sigma model coupling constant (4.9) one can check that the integral in (4.9) reduces to a total derivative and given by the flux of the string determined by $f_{3}(0)=1$. Namely, ${ }^{11}$

$$
\begin{aligned}
I & \equiv \int_{0}^{\infty} r d r\left\{\left(\frac{d}{d r} \rho(r)\right)^{2}+\frac{1}{r^{2}} f_{3}^{2}(1-\rho)^{2}\right. \\
& \left.+g_{2}^{2}\left[\frac{\rho^{2}}{2}\left(\phi_{1}^{2}+\phi_{2}^{2}\right)+(1-\rho)\left(\phi_{1}-\phi_{2}\right)^{2}\right]\right\} \\
& =\int_{0}^{\infty} d r\left(-\frac{d}{d r} f_{3}\right)=1,
\end{aligned}
$$

\footnotetext{
${ }^{11}$ The numerical result of R. Auzzi mentioned above was $I=1.00008$.
} 
where we use the first order equations (3.19) for the profile functions of the string. Returning to the $C P^{1}$ model (4.8) we conclude that the sigma model coupling $\beta$ does not depend on the ratio of $\mathrm{U}(1)$ and $\mathrm{SU}(2)$ coupling constants and is given by

$$
\beta=\frac{2 \pi}{g_{2}^{2}} .
$$

The two-dimensional coupling constant is determined by the four-dimensional nonAbelian coupling. As we will see later, this fact is very important for our interpretation of confined monopoles as sigma-model kinks.

In summary, the effective world-sheet theory describing dynamics of the string orientational zero modes is the celebrated $O(3)$ sigma model (which is the same as $\left.C P^{1}\right)$. The symmetry of this model reflects the presence of the global $\mathrm{SU}(2)_{C+F}$ symmetry in the microscopic theory. The coupling constant of this sigma model is determined by minimization of the action (4.9) for the profile function $\rho$. The minimal value of $I$ is unity. Clearly, Eq. (4.8) describes the low-energy limit. In principle, the zero-mode interaction has higher derivative corrections which run in powers of

$$
\left(g_{2} \sqrt{\xi}\right)^{-1} \partial_{n}
$$

where $g_{2} \sqrt{\xi}$ gives the order of magnitude of masses of the gauge/quark multiplets in our microscopic $\mathrm{SU}(2) \times \mathrm{U}(1)$ theory. The sigma model (4.8) is adequate at scales below $g_{2} \sqrt{\xi}$ where higher-derivative corrections are negligibly small.

The very same scale, $\left(g_{2} \sqrt{\xi}\right)^{-1}$, determines the thickness of the strings we deal with. In other words, the effective sigma model (4.8) is applicable at scales below the inverse string thickness which, thus, plays the role of an ultraviolet (UV) cutoff for the model (4.8).

\section{$4.2(1+1)$-dimensional $C P^{1}$ model}

Let us discuss the theory on the string world sheet as it emerges after factoring out (super)translational moduli. As was mentioned, the solution of the string BPS condition is in fact a two-parametric family of solutions parametrized by $\vec{n}$ with the constraint $\vec{n}^{2}=1$. The target space of the bosonic moduli is $\mathrm{SU}(2) / \mathrm{U}(1)$, the same as $C P^{1}$.

Since it is also endowed with four supercharges, the world-sheet theory must be $\mathcal{N}=2$ two-dimensional $C P^{1}$ sigma model. In Sect. 4.4 we will explicitly construct four fermion zero modes in the microscopic theory (not counting supertranslational) which match two bosonic zero modes associated with the color-magnetic flux rotation in the $\mathrm{O}(3)$ group space, Fig. 6. This will essentially conclude the proof that the world-sheet theory is the $C P^{1}$ sigma model. Let us briefly review the properties of this model. 
The $\mathcal{N}=2$ two-dimensional $C P^{1}$ model has the following action (e.g. [35], Sect. $6)$ :

$$
S_{C P(1)}=\beta \int d^{2} x\left\{\frac{1}{2}\left(\partial_{k} n^{a}\right)^{2}+\frac{i}{2} \bar{\chi}^{a} \gamma_{k} \partial_{k} \chi^{a}+\frac{1}{8}(\bar{\chi} \chi)^{2}\right\}
$$

where the $\gamma$ matrices in $(1+1)$ dimensions are defined as ${ }^{12}$

$$
\gamma_{0}=\tau_{1}, \quad \gamma_{3}=-\tau_{2}
$$

while $\chi^{a}$ is a real two component Majorana fermion field $\left(\bar{\chi}=\chi \gamma_{0}\right)$. It is subject to the constraint

$$
\chi^{a} n^{a}=0
$$

therefore, in fact, we have four real fermion components, as expected. Note that $\beta$ is related to the conventional coupling constant of the $C P^{1}$ model as $\beta=1 / g_{C P(1)}^{2}$, and the loop expansion runs in powers of $g_{C P(1)}^{2} / \pi$. Given Eq. (4.13) we see that that the loop expansion parameter is $g_{2}^{2} /\left(2 \pi^{2}\right)$ which coincides with the loop expansion parameter in the microscopic theory.

In the holomorphic representation upon the stereographic projection the Lagrangian of the $C P^{1}$ model (4.15) becomes

$$
\begin{aligned}
& \mathcal{L}_{C P(1)}=G\left\{\partial_{k} \bar{w} \partial_{k} w+\frac{i}{2}\left(\bar{\Psi}_{L} \overleftrightarrow{\partial}_{R} \Psi_{L}+\bar{\Psi}_{R} \overleftrightarrow{\partial}_{L} \Psi_{R}\right)\right. \\
& \left.-\frac{i}{\zeta}\left[\bar{\Psi}_{L} \Psi_{L}\left(\bar{w} \overleftrightarrow{\partial}_{R} w\right)+\bar{\Psi}_{R} \Psi_{R}\left(\bar{w} \overleftrightarrow{\partial_{L}} w\right)\right]-\frac{2}{\zeta^{2}} \bar{\Psi}_{L} \Psi_{L} \bar{\Psi}_{R} \Psi_{R}\right\} \\
& +\frac{i \theta}{2 \pi} \frac{1}{\zeta^{2}} \varepsilon^{m k} \partial_{m} \bar{w} \partial_{k} w
\end{aligned}
$$

where $G$ is the metric on the target space,

$$
G \equiv 2 \beta \frac{1}{(1+w \bar{w})^{2}},
$$

and

$$
\zeta \equiv 1+w \bar{w}
$$

(It is useful to note that the Ricci tensor $R=2 \zeta^{-2}$.) For completeness we also included the vacuum-angle term, see the last term in Eq. (4.18). Furthermore, the fermion field is a two-component Dirac spinor

$$
\Psi=\left(\begin{array}{c}
\Psi_{R} \\
\Psi_{L}
\end{array}\right)
$$

\footnotetext{
${ }^{12}$ This is a "Euclidean" notation, see footnote to Eq. (3.3).
} 
Finally, the bars over $w$ and $\Psi_{L, R}$ denote Hermitean conjugation.

The sigma model (4.15) or (4.18) is asymptotically free [36]; at large distances (low energies) it gets into the strong coupling regime. The corresponding GellMann-Low function is one-loop, and the running coupling constant as a function of the energy scale $E$ is given by

$$
4 \pi \beta=2 \ln \left(\frac{E}{\Lambda_{2 D}}\right)
$$

where $\Lambda_{2 D}$ is the dynamical scale of the sigma model; a related definition of this scale is given below in Eq. (4.27). As was mentioned previously, the ultraviolet cut-off of the sigma model at hand is determined by $g_{2} \sqrt{\xi}$. At this UV cut-off scale Eq. (4.13) holds. Hence,

$$
\Lambda_{2 D}^{2}=\xi e^{-\frac{8 \pi^{2}}{g_{2}^{2}}}=\Lambda^{2},
$$

where we take into account Eq. (3.9) for the dynamical scale $\Lambda$ of the $\mathrm{SU}(2)$ factor of the microscopic theory. Note that in the microscopic theory per se, because of the VEV's of the squark fields, the coupling constant is frozen at $g_{2} \sqrt{\xi}$; there are no logarithms below this scale. The logarithms of the macroscopic theory take over. Moreover, the dynamical scales of the microscopic and microscopic theories turn out to be the same! We will explain the reason why the dynamical scale of the $(1+1)$ dimensional effective theory on the string world sheet equals that of the $\mathrm{SU}(2)$ factor of the (3+1)-dimensional gauge theory later, in Sect. 7 .

The superalgebra induced by four supercharges of the world-sheet theory is as follows:

$$
\begin{aligned}
\left\{\bar{Q}_{L} Q_{L}\right\} & =(H+P), \quad\left\{\bar{Q}_{R} Q_{R}\right\}=(H-P) ; \\
\left\{\bar{Q}_{R} Q_{L}\right\} & =\frac{1}{\pi} \int d z \partial_{z}\left(\zeta^{-2} \bar{\Psi}_{R} \Psi_{L}\right), \\
\left\{\bar{Q}_{L} Q_{R}\right\} & =\frac{1}{\pi} \int d z \partial_{z}\left(\zeta^{-2} \bar{\Psi}_{L} \Psi_{R}\right) .
\end{aligned}
$$

with all other anticommutators vanishing. Here $(H, P)$ is the energy-momentum operator. Equations (4.25) and (4.26) present a quantum anomaly derived in Ref. [20]. These anticommutators vanish at the classical level. The above anomaly is similar (and, in fact, related) to that in $\mathcal{N}=1$ supersymmetric gluodynamics [37]. Its occurrence is crucial for self-consistency of matching of the underlying microscopic theory with the macroscopic description provided by Eq. (4.18). The fact that the anomaly does take place can be viewed as a test that we are on the right track.

As well-known, two-dimensional $C P^{1}$ model possesses two vacua labeled by the bifermion order parameter,

$$
\left\langle\zeta^{-2} \bar{\Psi}_{R} \Psi_{L}\right\rangle= \pm \Lambda_{C P(1)} e^{i \theta / 2} .
$$


The distinct vacua of the world-sheet effective theory, in the language of the microscopic theory, describe two distinct strings. The physical meaning of this distinction will be revealed shortly. The dynamical scale $\Lambda_{2 D}$ defined in Eq. (4.22) is of the order of $\Lambda_{C P(1)}$. More exactly, $\Lambda_{2 D}=e \Lambda_{C P(1)}$.

We will interrupt here our discussion of the superalgebra in the macroscopic theory, with the intention to return to it later, in Sect. 6.

\subsection{Unequal quark mass terms; $C P^{1}$ with the twisted mass}

The fact that we have two distinct vacua in the world-sheet theory - two distinct strings - is not quite intuitive in the above consideration. This is understandable. At the classical level the $\mathcal{N}=2$ two-dimensional sigma model has a continuous vacuum manifold $S_{2}$. This is in one-to-one correspondence with continuously many strings parametrized by $\vec{n}$. The continuous degeneracy is lifted only upon inclusion of quantum effects that occur (in the sigma model) at strong coupling. Gone with this lifting is the moduli nature of the fields $n^{a}$. They become massive. This is difficult to grasp.

To facilitate contact between the microscopic and macroscopic theories, it is instructive to start from a deformed microscopic theory so that the string moduli are lifted already at the classical level. Then the origin of the two-fold degeneracy of the non-Abelian strings become transparent. This will help us understand, in an intuitive manner, other features listed above. After this understanding is achieved, nothing prevents us from returning to our case of strings with non-Abelian moduli at the classical level, by smoothly suppressing the moduli-breaking deformation. The two-fold degeneracy will remain intact as it follows from the Witten index [38].

Thus, let us drop the assumption $m_{1}=m_{2}$ and introduce a small mass difference. We will still assume that

$$
\mu \equiv m_{1}-m_{2}>0 .
$$

At $m_{1} \neq m_{2}$ the flavor (global) SU(2) symmetry of the microscopic theory is explicitly broken down to $\mathrm{U}(1)$ (corresponding to rotations around the third axis in the $\mathrm{O}(3)$ group space). Correspondingly, the moduli of the non-Abelian string are lifted, since the vector $\vec{n}$ gets fixed in the position pointing in the third direction, $\vec{n}=\{0,0, \pm 1\}$. These are the $(1,0)$ and $(0,1)$ strings, respectively, see Sect. 3.1. If $\mu \ll \sqrt{\xi}$ the set of parameters $n^{a}$ becomes quasimoduli, as is clear from Fig. 7 .

Now, our aim is to derive the effective two-dimensional theory on the string world sheet for the case of unequal quark masses, when $\mathrm{SU}(2) \times \mathrm{U}(1)$ gauge theory is broken down to $\mathrm{U}(1) \times \mathrm{U}(1)$, assuming that $\mu$ is small. As was discussed in Sect. 3.1, the bona fide solutions of first-order equations (3.19) (and, hence, the equations of motion) with the minimal windings are the $(1,0)$ and $(0,1)$ strings. The solution for the $(1,0)$ string is given by $(3.18)$ while the solution for the $(0,1)$ string can be 
obtained from the one in Eq. (3.18) by the replacement

$$
f_{3} \rightarrow-f_{3}
$$

and

$$
e^{i \alpha} \phi_{1} \leftrightarrow \phi_{2}
$$

However, at small $\mu$ we can still introduce the orientational quasi-moduli $n^{a}$. In terms of the effective two-dimensional theory on the string world sheet $\mu \neq 0$ leads to a shallow potential for the quasi-moduli $n^{a}$. The two minima of the potential at $n=\{0,0, \pm 1\}$ correspond to two bona fide solutions for the $(1,0)$ and $(0,1)$ strings.

Let us derive this potential. To this end we start from the expression for the non-Abelian string in the singular gauge (3.33) parametrized by moduli $n^{a}$ and substitute it in the action (3.3). The only modification that we actually have to make is to supplement our ansatz (3.33) by that for the adjoint scalar field $a^{a}$; the neutral scalar field $a$ will stay fixed at its vacuum expectation value $a=-\sqrt{2} m$.

At large $r$ the field $a^{a}$ tends to its VEV directed along the third axis in the color space and is given by Eq. (3.5). At the same time, at $r=0$ it must be directed along the vector $n^{a}$. The reason for this behavior is easy to understand. The kinetic term for $a^{a}$ in Eq. (3.3) contains the commutator term of the adjoint scalar and the gauge potential. The gauge potential is singular at the origin, as is seen from Eq. (3.33). This implies that $a^{a}$ must be directed along $n^{a}$ at $r=0$. Otherwise, the string tension would become divergent. The following ansatz for $a^{a}$ ensures this behavior:

$$
a^{a}=-\frac{\mu}{\sqrt{2}}\left[\delta^{a 3} b+n^{a} n^{3}(1-b)\right] .
$$

Here we introduced a new profile function $b(r)$ which, as usual, will be determined from a minimization procedure. Note that at $n^{a}=(0,0, \pm 1)$ the field $a^{a}$ is given by its VEV, as expected. The boundary conditions for the function $b(r)$ are

$$
b(\infty)=1, \quad b(0)=0
$$

Substituting Eq. (4.29) in conjunction with (3.33) in the action (3.3) we get the potential

$$
V_{C P(1)}=\gamma \int d^{2} x \frac{\mu^{2}}{2}\left(1-n_{3}^{2}\right),
$$

where $\gamma$ is presented by the integral

$$
\begin{aligned}
\gamma & =\frac{2 \pi}{g_{2}^{2}} \int_{0}^{\infty} r d r\left\{\left(\frac{d}{d r} b(r)\right)^{2}+\frac{1}{r^{2}} f_{3}^{2} b^{2}+\right. \\
& \left.+g_{2}^{2}\left[\frac{1}{2}(1-b)^{2}\left(\phi_{1}^{2}+\phi_{2}^{2}\right)+b\left(\phi_{1}-\phi_{2}\right)\right]\right\} .
\end{aligned}
$$


Here two first terms in the integrand come from the kinetic term of the adjoint scalar field $a^{a}$ while the term in the square brackets comes from the last term in the action (3.3).

Minimization with respect to $b(r)$, with the constraint (4.30), yields

$$
b(r)=1-\rho(r)=\frac{\phi_{1}}{\phi_{2}}(r)
$$

cf. Eqs. (4.9), (4.11). Thus, $\gamma=I \times 2 \pi /\left(g_{2}^{2}\right)=2 \pi /\left(g_{2}^{2}\right)$. We see that the normalization integrals are the same for both, the kinetic and the potential terms in the world-sheet sigma model, $\gamma=\beta$. As a result we arrive at the following effective theory on the string world sheet:

$$
S_{C P(1), \mu}=\beta \int d^{2} x\left\{\frac{1}{2}\left(\partial_{k} n^{a}\right)^{2}+\frac{\mu^{2}}{2}\left(1-n_{3}^{2}\right)\right\} .
$$

This is the only functional form that allows $\mathcal{N}=2$ completion. ${ }^{13}$

The fact that we obtain this form shows that our ansatz is fully adequate. The informative aspect of the procedure is (i) the confirmation of the ansatz and (ii) constructive calculation of the constant in front of $\left(1-n_{3}^{2}\right)$ in terms of the microscopic parameters. The mass-splitting parameter $\Delta m$ of the microscopic theory exactly coincides with the twisted mass $\mu$ of the macroscopic model.

As was already mentioned this sigma model gives an effective description of our string at low energies, i.e. energies much lower than the inverse string thickness. Typical momenta in the theory (4.34) are of the order of $\mu$. Therefore, for the action (4.34) to be applicable we must impose the condition

$$
|\Delta m| \ll g_{2} \sqrt{\xi} .
$$

The $C P^{1}$ model (4.34) has two vacua located at $n^{a}=(0,0, \pm 1)$, see Fig. 7. Clearly these two vacua correspond to two elementary strings: $(1,0)$ and $(0,1)$, respectively.

Upon stereographic projection the action (4.34) takes the form

$$
S_{\mathrm{CP}(1), \mu}=\int d^{2} x G\left\{\partial_{k} \bar{w} \partial_{k} w+\mu^{2}|w|^{2}\right\},
$$

where $G$ is given in Eq. (4.19). We pause here to make a few remarks regarding the sigma model with $\mu \neq 0$.

First and foremost, Eq. (4.36) is the bosonic part of an $\mathcal{N}=2$ two-dimensional sigma model [39] which is usually referred to as the $C P^{1}$ model with the twisted mass. This is a generalization of the massless $C P^{1}$ model which preserves four supercharges. As we know, the BPS nature of the strings under consideration does require

\footnotetext{
${ }^{13}$ Note, that although the global $\mathrm{SU}(2)_{C+F}$ is broken by $\Delta m$, the extended $\mathcal{N}=2$ supersymmetry is not.
} 
the world-sheet theory to have four supercharges. Crucial for the construction of the twisted-mass $\mathcal{N}=2$ model is the fact that the target space of the $C P^{1}$ model has isometries. One can exploit the isometries to introduce the $\mathcal{N}=2$ supersymmetric mass term $\mu$, namely,

$$
\Delta_{\mu} \mathcal{L}_{\mathrm{CP}(1)}=G\left\{|\mu|^{2} w \bar{w}+i \frac{1-w \bar{w}}{\zeta}\left(\mu \bar{\Psi}_{L} \Psi_{R}-\bar{\mu} \bar{\Psi}_{R} \Psi_{L}\right)\right\},
$$

to be added to Eq. (4.18). Here $\zeta$ is defined in Eq. (4.20). Generically speaking, $\mu$ is a complex parameter. Certainly, one can always eliminate the phase of $\mu$ by a chiral rotation of the fermion fields. Due to the chiral anomaly, this will lead to a shift of the vacuum angle $\theta$. In what follows we will keep $\mu$ real and positive and ignore $\theta$ unless stated to the contrary. The U(1)-invariant scalar potential term in the holomorphic representation is

$$
V_{C P(1), \mu}=\mu^{2} G \bar{w} w
$$

If $\mu^{2} \gg \Lambda_{C P(1)}^{2}$, the classical description is fully applicable. As we already explained, two minima of $V_{C P(1), \mu}$, at $w=0$ and $w=\infty$, correspond to the $(1,0)$ and $(0,1)$ strings of the four-dimensional theory (Fig. 7).

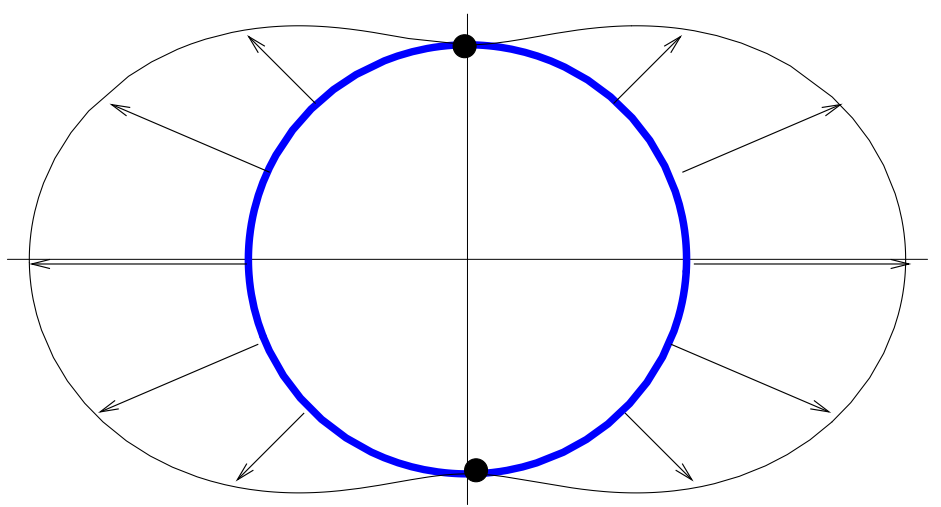

Figure 7: Meridian slice of the target space sphere (thick solid line). Arrows present the scalar potential (4.38), their length being the strength of the potential. Two vacua of the model are denoted by closed circles.

Now, after this lengthy digression, we can return to the limit $\Delta m \rightarrow 0$. The quasiclassical treatment of the world-sheet theory applies no longer since the world-sheet theory gets into the strong coupling regime, but we still have two vacua (Witten's 
index!). These two vacua differ from each other by the expectation value of the chiral bifermion operator (4.27), see e.g. [35]. At strong coupling $(\mu=0)$ the chiral condensate is the order parameter. The $C P^{1}$ model has a discrete $Z_{4}$ symmetry, a remnant of the anomalous $\mathrm{U}(1)$ chiral symmetry. The condensate (4.27) breaks it down to $Z_{2}$; hence, the two-fold degeneracy.

The physics of the model becomes more transparent in the mirror representation [40]. In this representation one describes the $C P^{1}$ model in terms of the Coulomb gas of instantons to prove its equivalence to a sine-Gordon theory. The $C P^{1}$ model (4.15) is dual to the following $\mathcal{N}=2$ sine-Gordon model [40]:

$$
S_{\mathrm{SG}}=\int d^{2} x d^{2} \theta d^{2} \bar{\theta} \beta^{-1} \bar{Y} Y+\left\{\frac{\Lambda_{C P(1)}}{2 \pi} \int d^{2} x d^{2} \theta \cosh Y+\text { h.c. }\right\} .
$$

Here the last term is a dual instanton-induced superpotential. The scalar potential of this sine-Gordon theory is

$$
V_{\mathrm{SG}}=\frac{\beta}{4 \pi^{2}} \Lambda_{C P(1)}^{2}|\sinh y|^{2},
$$

which has two minima, at $y=0$ and $y= \pm i \pi$. The target space of the mirror model is the cylinder depicted in Fig. 8a. That is why the points $y=i \pi$ and $y=-i \pi$ must be identified; they present one and the same vacuum. In Fig. $8 \mathrm{~b}$ this identification means gluing the lines $A$ and $B$. The straight lines in Fig. 8b passing through the points $y=0$ and $y= \pm i \pi$ are the lines on which the $\cosh Y$ superpotential is real. Since the imaginary part of the superpotential must vanish on the solutions of the BPS equations starting (ending) on the vacuum points, the solution trajectories can lie only on the above lines of "reality" of superpotential.

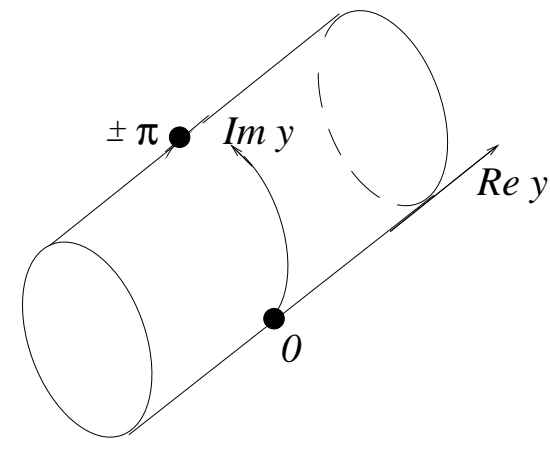

(a)

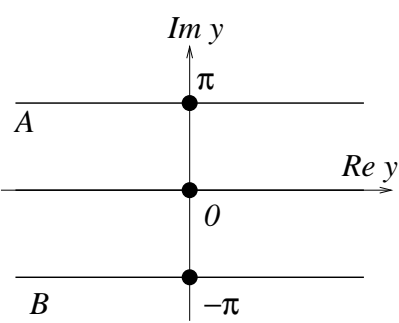

(b)

Figure 8: The target space of the mirror model and the trajectories of the BPS solutions interpolating between two vacua of the model. The vacua are denoted by closed circles. The lines $A$ and $B$ in Fig. $b$ must be identified. 
This mirror model explicitly exhibits a mass gap of the order of $\Lambda_{C P(1)}$. It shows that there are no Goldstone bosons (corresponding to the absence of the spontaneous breaking of of the microscopic theory $\mathrm{SU}(2)_{C+F}$ on the string). This means, in turn, that the string orientation vector $n^{a}$ has no particular direction, it is smeared all over. The strings we deal here are genuinely non-Abelian. Two degenerate strings of the microscopic theory corresponding to two degenerate vacua of the theory (4.18) are two "elementary" non-Abelian strings of the $\Delta m=0$ theory which form a doublet of $\mathrm{SU}(2)_{C+F}$. They are not the $(1,0)$ and $(0,1)$ strings of the quasiclassical $\mathrm{U}(1) \times \mathrm{U}(1)$ theory since the vector $n^{a}$ has no particular direction.

The mirror model also demonstrates the occurrence of two distinct kinks interpolating between the two vacua of the model. (More precisely, since each kink has two fermion zero modes, we should speak of two distinct dimension-two supermultiplets.) The two solutions are

$$
y= \pm\left\{\frac{\pi}{2}+\arcsin \left[\tanh m_{y} z\right]\right\}, \quad m_{y}=\beta \Lambda_{C P(1)} /(2 \pi) .
$$

The kink doubling is in full accord with the fact that the Cecotti-Fendley-IntriligatorVafa (CFIV) index [41] of the $C P(1)$ model equals two.

\subsection{Fermion zero modes of the string}

In this section we use supersymmetry to explicitly construct the fermion zero modes - those not associated with supertranslations - and show that they reproduce the fermion part of $\mathcal{N}=2$ two-dimensional $C P^{1}$ model on the string world sheet. We will set $m_{1}=m_{2}$ and work with the string solution (3.33) in the singular gauge.

Our string solution is $1 / 2$ BPS-saturated. This means that four supercharges, out of eight of the four-dimensional theory, act trivially on the string solution (3.33). The remaining four supercharges generate four fermion zero modes which are called supertranslational modes because they are superpartners to two translational zero modes. The corresponding four fermionic moduli are superpartners to the coordinates $x_{0}$ and $y_{0}$ of the string center. The supertranslational fermion zero modes were found in Ref. [23]. As a matter of fact, they were found for the U(1) ANO string in $\mathcal{N}=2$ theory but the transition to the model at hand is absolutely straightforward. We will not dwell on this procedure here.

Instead, we will focus on four additional fermion zero modes which arise only for the non-Abelian string (at $m_{1}=m_{2}$ ). They are superpartners of the bosonic orientational moduli $n^{a}$; therefore, we will refer to these modes as superorientational.

To obtain these modes in the simplest and fastest way we designed a special strategy which is outlined below. We start from the supersymmetry transformations for the fermion fields in the microscopic theory,

$$
\delta \lambda^{f \alpha}=\frac{1}{2}\left(\sigma_{\mu} \bar{\sigma}_{\nu} \epsilon^{f}\right)^{\alpha} F_{\mu \nu}+\epsilon^{\alpha p} D^{m}\left(\tau^{m}\right)_{p}^{f}+\ldots,
$$




$$
\begin{aligned}
\delta \lambda^{a f \alpha} & =\frac{1}{2}\left(\sigma_{\mu} \bar{\sigma}_{\nu} \epsilon^{f}\right)^{\alpha} F_{\mu \nu}^{a}+\epsilon^{\alpha p} D^{a m}\left(\tau^{m}\right)_{p}^{f}+\ldots, \\
\delta \overline{\tilde{\psi}}_{\dot{\alpha}}^{k A} & =i \sqrt{2} \bar{\nabla}_{\dot{\alpha} \alpha} q_{f}^{k A} \epsilon^{\alpha f}+\cdots \\
\delta \bar{\psi}_{\dot{\alpha} A k} & =i \sqrt{2} \bar{\nabla}_{\dot{\alpha} \alpha} \bar{q}_{f A k} \epsilon^{\alpha f}+\cdots .
\end{aligned}
$$

Here $\lambda^{f \alpha}$ and $\lambda^{a f \alpha}$ are the fermions from the $\mathcal{N}=2$ vector supermultiplets of the $\mathrm{U}(1)$ and $\mathrm{SU}(2)$ factors, respectively, while $\psi^{k A}$ and $\tilde{\psi}_{A k}$ are the fermion partners of the squark fields $q^{k A}$ and $\tilde{q}_{A k}$ in the quark hypermultiplets. Moreover, $f=1,2$ is the $\mathrm{SU}(2)_{R}$ index, $\alpha=1,2$ is the spinor index, $k=1,2$ is the color index, and $A=1,2$ is the flavor index. The parameters of SUSY transformations in the microscopic theory are denoted as $\epsilon^{\alpha f}$. Furthermore, the $D$ terms in Eq. (4.41) are

$$
D^{1}+i D^{2}=i \frac{g_{1}^{2}}{2}\left(\operatorname{Tr}|\Phi|^{2}-2 \xi\right), \quad D^{3}=0
$$

for the $\mathrm{U}(1)$ field, and

$$
D^{a 1}+i D^{a 2}=i \frac{g_{2}^{2}}{2} \operatorname{Tr}\left(\Phi^{\dagger} \tau^{a} \Phi\right), \quad D^{a 3}=0
$$

for the $\mathrm{SU}(2)$ field. The dots in (4.41) stand for terms involving the adjoint scalar fields which vanish on the string solution (at $m_{1}=m_{2}$ ) because the adjoint fields are given by their vacuum expectation values (3.7).

In Ref. [23] it was shown that the four supercharges selected by the conditions

$$
\epsilon^{12}=-\epsilon^{11}, \quad \epsilon^{21}=\epsilon^{22}
$$

act trivially on the BPS string. Now, to generate the superorientational fermion zero modes we use the following method. We assume that the orientational moduli $n^{a}$ in the string solution (3.33) have a slow dependence on the world-sheet coordinates $x_{0}$ and $x_{3}$ (or $t$ and $z$ ), as in Sect. 4.1. Then the four supercharges selected by the conditions (4.44) no longer act trivially. Instead, their action now gives fermion fields proportional to the $x_{0}$ and $x_{3}$ derivatives of $n^{a}$. This is exactly what one expects from the residual $\mathcal{N}=2$ supersymmetry in the world-sheet theory. The above four supercharges generate the world-sheet supersymmetry in the $\mathcal{N}=2$ twodimensional $C P^{1}$ model. We use this world-sheet supersymmetry to re-express the fermion fields obtained upon the action of these four supercharges in terms of the $(1+1)$-dimensional fermions. This will give us the superorientational fermion zero modes.

After this brief outline we can proceed to the implementation of the procedure. We substitute the string solution (3.33) in (4.41) assuming that $\epsilon^{\alpha f}$ are subject to 
the constraints (4.44) and the moduli $n^{a}$ have a slow dependence on the world-sheet coordinates. Then we get

$$
\begin{aligned}
\bar{\psi}_{A k \dot{2}} & =i\left(\tau^{a}\right)_{A k}\left\{\left[\left(\partial_{0}-i \partial_{3}\right) n^{a}\right]\left(\phi_{1}-\phi_{2}\right)\left(1-\frac{\rho}{2}\right)\right. \\
& \left.-i \varepsilon^{a b c} n^{b}\left[\left(\partial_{0}-i \partial_{3}\right) n^{c}\right] \frac{\rho}{2}\left(\phi_{1}+\phi_{2}\right)\right\} \epsilon^{22}, \\
\overline{\tilde{\psi}}_{\dot{1}}^{k A} & =i\left(\tau^{a}\right)^{k A}\left\{\left[\left(\partial_{0}+i \partial_{3}\right) n^{a}\right]\left(\phi_{1}-\phi_{2}\right)\left(1-\frac{\rho}{2}\right)\right. \\
& \left.+i \varepsilon^{a b c} n^{b}\left[\left(\partial_{0}+i \partial_{3}\right) n^{c}\right] \frac{\rho}{2}\left(\phi_{1}+\phi_{2}\right)\right\} \epsilon^{11}, \\
\bar{\psi}_{A k \dot{1}} & =0, \quad \overline{\tilde{\psi}}_{\dot{2}}^{k A}=0, \\
\lambda^{a 22} & =-\frac{x_{1}+i x_{2}}{r^{2}}\left\{\left[\left(\partial_{0}+i \partial_{3}\right) n^{a}\right] f_{3}(1-\rho)+i \varepsilon^{a b c} n^{b}\left[\left(\partial_{0}+i \partial_{3}\right) n^{c}\right] r \frac{d}{d r} \rho\right\} \epsilon^{11}, \\
\lambda^{a 11} & =-\frac{x_{1}-i x_{2}}{r^{2}}\left\{\left[\left(\partial_{0}-i \partial_{3}\right) n^{a}\right] f_{3}(1-\rho)-i \varepsilon^{a b c} n^{b}\left[\left(\partial_{0}-i \partial_{3}\right) n^{c}\right] r \frac{d}{d r} \rho\right\} \epsilon^{22}, \\
\lambda^{12} & =\lambda^{11}, \quad \lambda^{21}=-\lambda^{22} .
\end{aligned}
$$

Let us compare these transformations with the supersymmetry transformations on the string world sheet. For the model (4.15) they are

$$
\delta \chi^{a}=i \sqrt{2}\left[\left(\gamma_{k} \partial_{k} n^{a}\right) \varepsilon+\varepsilon^{a b c} n^{b}\left(\gamma_{k} \partial_{k} n^{c}\right) \eta\right],
$$

where $\varepsilon^{\alpha}$ is a real two-component parameter of the two-dimensional supersymmetry, while $\eta^{\alpha}$ is another such parameter $(\alpha=1,2)$, so that assembled together they form a full set of $\mathcal{N}=2$ transformations. If we rewrite Eq. (4.46) in components,

$$
\begin{aligned}
\delta \chi_{1}^{a} & =i \sqrt{2}\left[\left(\partial_{0}+i \partial_{3}\right) n^{a} \varepsilon_{2}+\varepsilon^{a b c} n^{b}\left(\partial_{0}+i \partial_{3}\right) n^{c} \eta_{2}\right], \\
\delta \chi_{2}^{a} & =i \sqrt{2}\left[\left(\partial_{0}-i \partial_{3}\right) n^{a} \varepsilon_{1}+\varepsilon^{a b c} n^{b}\left(\partial_{0}-i \partial_{3}\right) n^{c} \eta_{1}\right],
\end{aligned}
$$

and identify properly normalized parameters of the four-dimensional SUSY transformations (with the constraint (4.44)) in terms of $\varepsilon_{1,2}, \eta_{1,2}$, namely,

$$
\begin{aligned}
& \varepsilon_{2}+i \eta_{2}=\frac{1}{\sqrt{2}}\left(\epsilon^{11}-\epsilon^{12}\right)=\sqrt{2} \epsilon^{11}, \\
& \varepsilon_{1}-i \eta_{1}=\frac{1}{\sqrt{2}}\left(\epsilon^{22}+\epsilon^{21}\right)=\sqrt{2} \epsilon^{22},
\end{aligned}
$$


we can express the derivatives of $n^{a}$ in Eq. (4.45) in terms of $\chi_{1}^{a}$ and $\chi_{2}^{a}$, thus obtaining the zero modes of the quark and gluino fields in terms of the four superorientational moduli. In this way we arrive at

$$
\begin{aligned}
\bar{\psi}_{A k \dot{2}} & =\left(\frac{\tau^{a}}{2}\right)_{A k} \frac{1}{2 \phi_{2}}\left(\phi_{1}^{2}-\phi_{2}^{2}\right)\left[\chi_{2}^{a}+i \varepsilon^{a b c} n^{b} \chi_{2}^{c}\right] \\
\overline{\tilde{\psi}}_{\dot{1}}^{k A} & =\left(\frac{\tau^{a}}{2}\right)^{k A} \frac{1}{2 \phi_{2}}\left(\phi_{1}^{2}-\phi_{2}^{2}\right)\left[\chi_{1}^{a}-i \varepsilon^{a b c} n^{b} \chi_{1}^{c}\right], \\
\bar{\psi}_{A k \dot{1}} & =0, \quad \overline{\tilde{\psi}}_{\dot{2}}^{k A}=0 \\
\lambda^{a 22} & =\frac{i}{2} \frac{x_{1}+i x_{2}}{r^{2}} f_{3} \frac{\phi_{1}}{\phi_{2}}\left[\chi_{1}^{a}-i \varepsilon^{a b c} n^{b} \chi_{1}^{c}\right], \\
\lambda^{a 11} & =\frac{i}{2} \frac{x_{1}-i x_{2}}{r^{2}} f_{3} \frac{\phi_{1}}{\phi_{2}}\left[\chi_{2}^{a}+i \varepsilon^{a b c} n^{b} \chi_{2}^{c}\right] \\
\lambda^{12} & =\lambda^{11}, \quad \lambda^{21}=-\lambda^{22},
\end{aligned}
$$

where we use the solution (4.11) for the function $\rho$ to simplify the expressions for the profile functions of the fermion zero modes. Equation (4.49) is our final result for the superorientational fermion zero modes. Here the dependence on $x_{i}$ is encoded in the profile functions of the string, while $\chi_{\alpha}^{a}$ should be considered as constant Grassmann collective coordinates.

To conclude this section let us check ${ }^{14}$ that the zero modes above do produce the fermion part of the $\mathcal{N}=2$ two-dimensional $C P^{1}$ model (4.15). To this end we return to the usual assumption that the fermion collective coordinates $\chi_{\alpha}^{a}$ in Eq. (4.49) have an adiabatic dependence on the world sheet coordinates $x_{k}(k=0,3)$. This is quite similar to the procedure of Sect. 4.1. Substituting Eq. (4.49) in the kinetic terms of fermions in the microscopic theory,

$$
\int d^{4} x\left\{\bar{\psi}_{A} i \bar{\nabla} \psi^{A}+\overline{\tilde{\psi}}^{A} i \bar{\nabla} \tilde{\psi}_{A}+\frac{i}{g_{2}^{2}} \bar{\lambda}_{f}^{a} \bar{D} \lambda^{a f}\right\},
$$

and taking into account the derivatives of $\chi_{\alpha}^{a}$ with respect to the world-sheet coordinates, after some algebra we arrive at

$$
\beta \int d^{2} x\left\{\frac{1}{2} \bar{\chi}^{a} i \gamma_{n} \partial_{n} \chi^{a}\right\}
$$

where $\beta$ is given by the same integral (4.9) as for the bosonic kinetic term, see Eq. (4.8). We see that (4.51) exactly reproduces the kinetic term of the $(1+1)$ dimensional fermions in the $C P^{1}$ model (4.15).

\footnotetext{
${ }^{14}$ For simplicity, we restrict ourselves to terms in the action quadratic in the fermion fields.
} 


\section{Sigma-model kinks: monopoles of the micro- scopic theory}

Thus, we concluded our consideration of non-Abelian strings in the microscopic theory and derivation of the macroscopic world-sheet theory they induce. Our task in this section is the study and analysis of the BPS string junctions - the confined monopoles - both in the microscopic and macroscopic theories

A general picture is best inferred from consideration in the quasiclassical regime, namely, we start from the case $\Delta m \neq 0$ but subject to the constraint (4.35). This limit was studied in Ref. [6]. In the $C P^{1}$ model with the twisted mass there is a kink (a.k.a domain wall) interpolating between two vacua at the north and south poles, see Fig. 7. Two distinct strings of the microscopic theory are the two vacua of the macroscopic model, while the confined monopole is the $C P^{1}$ kink interpolating between them. We intend to back up this qualitative statement by quantitative data.

The $C P^{1}$ kink solution is easy to find in the explicit form. This is discussed in Sect. 5.3, as well as the occurrence of an "extra" collective coordinate. Kinks in the $\mathcal{N}=2$ two-dimensional sigma model with the twisted mass are exhaustively studied in the literature [4]. These kinks are 1/2 BPS saturated (i.e. preserve two supercharges). The corresponding collective coordinates are $z_{0}$ (a complexified position of the center) and two fermion moduli. We will say more on why $z_{0}$ gets complexified.

Now, if we send $\Delta m \rightarrow 0$ we still have two vacua in the $C P^{1}$ model, as was explained in Sect. 4.3, and do have kinks interpolating between them. This kink is best seen in the mirror description of the model. It interpolates between the two vacua of sine-Gordon potential (4.40). The kinks are counted in dimension-two supermultiplets. The Cecotti-Fendley-Intriligator-Vafa (CFIV) index [41] tells us that, in fact, we have two distinct kinks, albeit this number is not invariant under the variation of the twisted mass, see Sect. 5.3.7.

The $\Delta m=0$ kink should be interpreted as a "highly bound" monopole (it has multiplicity two as well) which realizes a junction of two "elementary" strings. However, the quantum numbers of this monopole are no longer $(1,-1)$. Similar to strings, it does not have definite Abelian charges in the limit $\Delta m \rightarrow 0$. It becomes a bona fide non-Abelian monopole of $\mathrm{SU}(2)$ representing the junction between two "elementary" non-Abelian strings associated with two quantum vacua of $C P^{1}$ model (Fig. 1, the right lower corner).

There are two features of the $\Delta m=0$ kinks that we can established on general grounds. First, their BPS saturated nature and Eqs. (4.25), (4.26) tell us that the kink mass is equal to [20]

$$
M_{k}=\frac{1}{\pi}\left|\Delta\left\langle\zeta^{-2} \bar{\Psi}_{R} \Psi_{L}\right\rangle\right|=\frac{2}{\pi} \Lambda_{C P(1)} .
$$


Second, the size of the kink (in the $z$ direction) is of the order $\sim \Lambda_{C P(1)}^{-1}$.

This means that the $\mathrm{SU}(2)$ monopole, although classically massless and infinitely spread in the limit $\Delta m \rightarrow 0$, in fact acquires a small but finite mass and finite size due to non-perturbative effects on the string world sheet. In this section we give a detailed quantitative evidence in favor of our identification of the $\mathrm{SU}(2)$ monopole as the $C P^{1}$ model kink. We start with the quasiclassical limit of non-zero $\Delta m$ considered by Tong [6] and then eventually arrive at the quantum limit $\Delta m \rightarrow 0$.

\subsection{Microscopic theory: first-order master equations}

In this section we derive the first-order equations for the $1 / 4$-BPS junction of the $(1,0)$ and $(0,1)$ strings in the quasiclassical limit

$$
\Lambda_{C P(1)} \ll \mu \ll g_{2} \sqrt{\xi}, \quad \mu=\Delta m .
$$

In this limit $\mu$ is small enough so we can use our effective low-energy description in terms of the $C P^{1}$ model (with the twisted mass). On the other hand, $\mu$ is much larger then the scale of $C P^{1}$ model, so the latter is in the weak coupling regime which allows one to apply the quasiclassical treatment.

The geometry of our junction is shown on Fig. 2b. Both strings are stretched along the $z$ axis. We assume that the monopole sits near the origin, the $(0,1)$-string is at negative $z$, while the $(1,0)$-string is at positive $z$. The perpendicular plane is parametrized by $x_{1}$ and $x_{2}$. What is sought for is a static solution of the BPS equations, with all relevant fields depending only on $x_{1}, x_{2}$ and $z$.

Ignoring the time variable we can represent the energy functional of our theory (3.3) as follows (Bogomolny representation [42]):

$$
\begin{aligned}
E & =\int d^{3} x\left\{\left[\frac{1}{\sqrt{2} g_{2}} F_{3}^{* a}+\frac{g_{2}}{2 \sqrt{2}}\left(\bar{\varphi}_{A} \tau^{a} \varphi^{A}\right)+\frac{1}{g_{2}} D_{3} a^{a}\right]^{2}\right. \\
& +\left[\frac{1}{\sqrt{2} g_{1}} F_{3}^{*}+\frac{g_{1}}{2 \sqrt{2}}\left(\left|\varphi^{A}\right|^{2}-2 \xi\right)+\frac{1}{g_{1}} \partial_{3} a\right]^{2} \\
& +\frac{1}{g_{2}^{2}}\left|\frac{1}{\sqrt{2}}\left(F_{1}^{* a}+i F_{2}^{* a}\right)+\left(D_{1}+i D_{2}\right) a^{a}\right|^{2} \\
& +\frac{1}{g_{1}^{2}}\left|\frac{1}{\sqrt{2}}\left(F_{1}^{*}+i F_{2}^{*}\right)+\left(\partial_{1}+i \partial_{2}\right) a\right|^{2} \\
& +\left|\nabla_{1} \varphi^{A}+i \nabla_{2} \varphi^{A}\right|^{2}
\end{aligned}
$$




$$
\left.+\left|\nabla_{3} \varphi^{A}+\frac{1}{\sqrt{2}}\left(a^{a} \tau^{a}+a+\sqrt{2} m_{A}\right) \varphi^{A}\right|^{2}\right\}
$$

plus surface terms. Following our conventions we assume the quark masses to be real implying that the vacuum expectation values of the adjoint scalar fields are real too. The surface terms mentioned above are

$$
E_{\text {surface }}=\xi \int d^{3} x F_{3}^{*}+\left.\sqrt{2} \xi \int d^{2} x\langle a\rangle\right|_{z=-\infty} ^{z=\infty}-\sqrt{2} \frac{\left\langle a^{3}\right\rangle}{g_{2}^{2}} \int d S_{n} F_{n}^{* 3},
$$

where the integral in the last term runs over a large two-dimensional sphere at $\vec{x}^{2} \rightarrow \infty$. The first term on the right-hand side is related to strings, the second to domain walls, while the third to monopoles (string junctions).

The Bogomolny representation (5.3) leads us to the following first-order equations:

$$
\begin{aligned}
& F_{1}^{*}+i F_{2}^{*}+\sqrt{2}\left(\partial_{1}+i \partial_{2}\right) a=0, \\
& F_{1}^{* a}+i F_{2}^{* a}+\sqrt{2}\left(D_{1}+i D_{2}\right) a^{a}=0, \\
& F_{3}^{*}+\frac{g_{1}^{2}}{2}\left(\left|\varphi^{A}\right|^{2}-2 \xi\right)+\sqrt{2} \partial_{3} a=0, \\
& F_{3}^{* a}+\frac{g_{2}^{2}}{2}\left(\bar{\varphi}_{A} \tau^{a} \varphi^{A}\right)+\sqrt{2} D_{3} a^{a}=0, \\
& \nabla_{3} \varphi^{A}=-\frac{1}{\sqrt{2}}\left(a^{a} \tau^{a}+a+\sqrt{2} m_{A}\right) \varphi^{A}, \\
& \left(\nabla_{1}+i \nabla_{2}\right) \varphi^{A}=0 .
\end{aligned}
$$

These are our master equations. Once these equations are satisfied the energy of the BPS object is given by Eq. (5.4).

Let us discuss the central charges (the surface terms) of the string, domain wall and monopole in more detail. Say, in the string case, the three-dimensional integral in the first term in Eq. (5.4) gives the length of the string times its flux. In the wall case, the two-dimensional integral in the second term in (5.4) gives the area of the wall times its tension. Finally, in the monopole case the integral in the last term in Eq. (5.4) gives the magnetic-field flux. This means that the first-order master equations (5.5) can be used to study strings, domain walls, monopoles and all their possible junctions.

It is instructive to check that the wall, the string and the monopole solutions, separately, satisfy these equations. For the domain wall this check was done in [2] where we used these equations to study the string-wall junctions. Let us consider 
the string solution. Then the scalar fields $a$ and $a^{a}$ are given by their VEV's. The gauge flux is directed along the $z$ axis, so that $F_{1}^{*}=F_{2}^{*}=F_{1}^{* a}=F_{2}^{* a}=0$. All fields depend only on the perpendicular coordinates $x_{1}$ and $x_{2}$. As a result, the first two equations and the fifth one in (5.5) are trivially satisfied. The third and the fourth equations reduce to the first two equations in Eq. (3.15). The last equation in (5.5) reduces to the last equation in (3.15).

Now, turn to the monopole solution. The 't Hooft-Polyakov monopole equations [19] arise from those in Eq. (5.5) in the limit $\xi=0$. Then all quark fields vanish, and Eq. (5.5) reduces to the standard first-order equations for the BPS 't Hooft-Polyakov monopole,

$$
F_{k}^{* a}+\sqrt{2} D_{k} a^{a}=0 .
$$

The $\mathrm{U}(1)$ scalar field $a$ is given by its VEV while the $\mathrm{U}(1)$ gauge field vanishes.

Now, Eq. (5.4) shows that the central charge of the $\mathrm{SU}(2)$ monopole is determined by $\left\langle a^{3}\right\rangle$ which is proportional to the quark mass difference. Thus, for the monopole on the Coulomb branch (i.e. with $\xi$ vanishing) Eq. (5.4) yields

$$
M_{m}=\frac{4 \pi \mu}{g_{2}^{2}} .
$$

This coincides, of course, with the Seiberg-Witten result [11] in the weak coupling limit. As we will see shortly, the same expression continues to hold even if $\mu \ll \sqrt{\xi}$ (provided that $\mu$ is still much larger than $\Lambda$ ). An explanation will be given in Sect. 7 .

We pause here to make a remark on the literature. The Abelian version of the first-order equations (5.5) were derived in Ref. [1] where they were used to find the 1/4 BPS-saturated solution for the wall-string junction. A subset of nonAbelian equations (5.5) in the $\mathrm{SU}(2) \times \mathrm{U}(1)$ theory was derived in [6] with the purpose of studying the junction of two elementary strings ("a confined monopole") at $\Delta m \neq 0$. We extensively exploit this construction too, as a reference point, while our main interest is the limit $\Delta m=0$. Non-Abelian equations (5.5) were derived and extensively used in the recent analysis [2] of the wall-string junctions for non-Abelian strings ending on a stack of domain walls.

\subsection{The string junction solution for $\sqrt{\xi} \gg \mu \gg \Lambda$}

Now we apply our master equations in order to find the junction of the $(0,1)$ and $(1,0)$ strings via the $(1,-1)$ monopole (see Fig. 1, the left lower corner) in the quasiclassical limit. We will show that the solution of the BPS equations (5.5) of the four-dimensional microscopic theory is determined by the kink solution in the two-dimensional sigma model (4.34).

To this end we will look for the solution of equations (5.5) in the following ansatz. Assume that the solution for the string junction is given, to the leading 
order in $\mu / \sqrt{\xi}$, by the same string configuration (3.33), and (4.29) which we dealt with previously, in Sect. 4.3 (in the case $\mu \neq 0$ ),

$$
\begin{aligned}
\Phi & =U\left(\begin{array}{cc}
\phi_{1}(r) & 0 \\
0 & \phi_{2}(r)
\end{array}\right) U^{-1}=\frac{1}{2}\left(\phi_{1}+\phi_{2}\right)+n^{a} \frac{\tau^{a}}{2}\left(\phi_{1}-\phi_{2}\right), \\
A_{i}^{a}(x) & =n^{a} \varepsilon_{i j} \frac{x_{j}}{r^{2}} f_{3}(r), \\
A_{i}(x) & =\varepsilon_{i j} \frac{x_{j}}{r^{2}} f(r), \\
A_{3}^{a} & =-\varepsilon^{a b c} n^{b}\left(\partial_{3} n^{c}\right) \rho(r), \quad A_{0}^{a}=0, \\
a^{a} & =-\frac{\mu}{\sqrt{2}}\left[\delta^{a 3}(1-\rho)+n^{a} n^{3} \rho\right], \quad a=-\sqrt{2} m,
\end{aligned}
$$

with $n^{a}$ slowly-varying functions of $z$, to be determined below, replacing the constant moduli vector $\vec{n}$. The ansatz for the gauge potentials $A_{3}^{a}$ and $A_{0}^{a}$ follows from Eqs. (4.1) and (4.4). As we have the (0,1)-string at $z \rightarrow-\infty$, the function $n^{a}(z)$ satisfy the boundary condition

$$
n^{a}(-\infty)=(0,0,-1)
$$

while

$$
n^{a}(\infty)=(0,0,1) .
$$

The latter condition ensures that we have the (1,0)-string at $z \rightarrow \infty$. The ansatz (5.8) corresponds to the non-Abelian string in which the vector $n^{a}$ slowly rotates from (5.9) at $z \rightarrow-\infty$ to (5.10) at $z \rightarrow \infty$. Now we will show that the representation (5.8) solves the master equations (5.5) provided the functions $n^{a}(z)$ are chosen in a special way.

Note that the first equation in (5.5) is trivially satisfied because the field $a$ is constant and $F_{1}^{*}=F_{2}^{*}=0$. The last equation reduces to the first two equations in (3.19) because it does not contain derivatives with respect to $z$ and, therefore, is satisfied for arbitrary functions $n^{a}(z)$. The same remark applies also to the third equation in Eq. (5.5), which reduces to the third equation in (3.19).

Now let us consider the fifth equation in Eq. (5.5). Substituting (5.8) in this equation and using expression (4.11) for $\rho$ we find that this equation is satisfied provided $n^{a}(z)$ are chosen to be the solutions of the equation

$$
\partial_{3} n^{a}=\mu\left(\delta^{a 3}-n^{a} n^{3}\right) .
$$

This equation, written in the holomorphic representation, is discussed in Sect. 5.3. 
By the same token, we can consider the second equation in (5.5). Upon substituting there the ansatz (5.8), it reduces to Eq. (5.11) too. Finally, consider the fourth equation in (5.5). One can see that in fact it contains an expansion in the parameter $\mu^{2} / \xi$. This means that the solution we have just built is not exact; it has corrections of the order of $O\left(\mu^{2} / \xi\right)$. To the leading order in this parameter the fourth equation in (5.5) reduces to the last equation in (3.19). In principle, one could go beyond the leading order. Solving the fourth equation in (5.5) in the nextto-leading order would allows one to determine $O\left(\mu^{2} / \xi\right)$ corrections to our solution (3.19). This goes beyond the scope of our current investigation.

Let us dwell on the meaning of Eq. (5.11). This equation is nothing but the equation for the kink in the $C P^{1}$ model (4.34). A thorough analysis of the $C P^{1}$ model kinks will be carried out in Sect. 5.3. Here we will limit ourselves to the Bogomolny completion of this model. The energy functional can be rewritten as

$$
E=\frac{\beta}{2} \int d z\left\{\left|\partial_{z} n^{a}-\mu\left(\delta^{a 3}-n^{a} n^{3}\right)\right|^{2}+2 \mu \partial_{z} n^{3}\right\}
$$

The above representation implies the first-order equation (5.11) for the BPS saturated kink. It also yields $2 \beta \mu$ for the kink mass.

Thus, we have demonstrated that the junction solution for the $(0,1)$ and $(1,0)$ strings is given by the non-Abelian string with a slowly varying orientation vector $n^{a}$. The variation of $n^{a}$ is described in terms of the kink solution of the $(1+1)$ dimensional $C P^{1}$ model with the twisted mass. This was expected.

In conclusion, we would like to match the masses of the four-dimensional monopole and two-dimensional kink. The string mass and that of the string junction is given by first and the last terms in the surface energy (5.4) (the second term vanishes). The first term obviously reduces to

$$
M_{\text {string }}=2 \pi \xi L
$$

i.e. proportional to the total string length $L$. Note that both the $(0,1)$ and $(1,0)$ strings have the same tension (3.14). The third term should give the mass of the $(1,-1)$ monopole. The surface integral in this term reduces to the flux of the $(1,0)$ string at $z \rightarrow \infty$ minus the flux of the $(0,1)$-string at $z \rightarrow-\infty$. The $F^{* 3}$ flux of the $(1,0)$-string is $2 \pi$ while the $F^{* 3}$ flux of the $(0,1)$-string is $-2 \pi$. Thus, taking into account Eq. (3.5), we get

$$
M_{m}=\frac{4 \pi}{g_{2}^{2}} \mu .
$$

Note, that although we discuss the monopole in the confinement phase at $|\Delta m| \ll$ $\sqrt{\xi}$ (which is a junction of two strings in this phase), nevertheless in terms of the $\mu$ and $g_{2}^{2}$ dependence its mass coincides with the result (5.7) for the unconfined monopole on the Coulomb branch (i.e. at $\xi=0$ ). There is no change in the 
monopole mass formula in the first three cases in Fig. 1. This is no accident there is a deep theoretical reason explaining the validity of the unified formula. A change occurs only in passing to the highly quantum regime depicted in the right lower corner of Fig. 1. We will discuss this regime shortly, see Eq. (5.18), while more details will be given in Sect. 7 .

Now let us compare (5.14) with the kink mass in the effective $C P^{1}$ model on the string world sheet. As was mentioned, the surface term in Eq. (5.12) gives

$$
M_{\mathrm{kink}}=2 \beta \mu=\frac{\mu}{\pi} \ln \frac{\mu}{\Lambda_{2 D}},
$$

where in the second equality we used Eq. (4.22) to express $\beta$ in terms of the dynamical scale parameter. Two remarks are in order here. First, expressing the two-dimensional coupling constant $\beta$ in terms of coupling constant of the microscopic theory, see Eq. (4.13), we obtain

$$
M_{\mathrm{kink}}=\frac{4 \pi}{g_{2}^{2}} \mu,
$$

thus verifying that the four-dimensional calculation of $M_{m}$ and the two-dimensional calculation of $M_{\text {kink }}$ yield the same,

$$
M_{m}=M_{\text {kink }} .
$$

Needless to say, this is in full accordance with the physical picture that emerged from our analysis, that the two-dimensional $C P^{1}$ model is nothing but the macroscopic description of the confined monopoles occurring in the four-dimensional microscopic Yang-Mills theory.

Technically the coincidence of the monopole and kink masses is based on the fact that the integral in the definition (4.9) of the sigma-model coupling $\beta$ is unity.

The second remark concerns the second equality in Eq. (5.15). In fact, using this form, one can get [4] a unified formula for $M_{\text {kink }}$ (and, hence, for $M_{m}$ ) describing the last two regimes in Fig. 1. To this end one replaces the logarithm

$$
\ln \frac{\mu}{\Lambda_{2 D}} \rightarrow\left|\frac{1}{2} \ln \frac{\sqrt{\mu^{2}+4 \Lambda_{C P(1)}^{2}}+\mu}{\sqrt{\mu^{2}+4 \Lambda_{C P(1)}^{2}}-\mu}-\sqrt{1+\frac{4 \Lambda_{C P(1)}^{2}}{\mu^{2}}}\right| .
$$

In the quasiclassical regime $\mu / \Lambda \gg 1$ the right-hand side and left-hand side coincide provided $\Lambda_{2 D}=e \Lambda_{C P(1)}$. If, on the other hand, $\mu \rightarrow 0$, combining Eqs. (5.15) and (5.18) we arrive at Eq. (5.1) for the kink mass. Using our identification of the fourdimensional monopole as a two-dimensional kink we then get the confined monopole mass,

$$
M_{m}=\frac{2}{\pi} \Lambda_{C P(1)}
$$

in the limit $\mu \rightarrow 0$. 


\subsection{More on kinks in the $C P^{1}$ model with the twisted mass}

Identification of the confined monopoles in four dimensions with the two-dimensional kinks yields an immediate bonus: all we know of the $C P^{1}$-model kinks can be rephrased in terms of the confined monopoles. The goal of this section is to briefly review $C P^{1}$-model kinks, with an eye on the parallel with the confined monopoles. Introductory data on the $C P^{1}$ model can be found in Sects. 4.2 and 4.3. We will heavily rely on the results of Refs. [4, 20]. The reader familiar with these results can proceed directly to Sect. 5.4. Among other topics, we will dwell on the role of the $\theta$ term and on "dyonic" kinks which are counterparts of "dyonic" confined monopoles of the microscopic theory.

\subsubsection{The $\theta$ term}

So far we chose $\mu$ to be real, and put $\theta=0$. Both requirements can and must be relaxed. Let us parametrize $\mu$ as

$$
\mu=|\mu| e^{i \omega}
$$

The chiral anomaly implies that neither $\omega$ not $\theta$ are separately observable. The physically observable phase combination is

$$
\theta_{\mathrm{eff}}=\theta+2 \omega
$$

Correspondingly, we can always eliminate $\theta$ by including it in the definition of the phase of $\mu$. Alternatively, we can always define $\mu$ to be real and positive, at a price of shifting the original $\theta$ by an appropriate amount, according to Eq. (5.21). Note that $\theta$ is defined $\bmod 2 \pi k$ where $k$ is an integer.

\subsubsection{Superalgebra at $\mu \neq 0$}

In the $C P^{1}$ model with the twisted mass

$$
\begin{aligned}
& \left\{Q_{L} \bar{Q}_{R}\right\}=-i \mu q_{\mathrm{U}(1)}-\mu \int d z \partial_{z} h+\frac{1}{\pi} \int d z \partial_{z}\left(\zeta^{-2} \bar{\Psi}_{R} \Psi_{L}\right) \\
& \left\{Q_{R} \bar{Q}_{L}\right\}=i \bar{\mu} q_{\mathrm{U}(1)}-\bar{\mu} \int d z \partial_{z} h+\frac{1}{\pi} \int d z \partial_{z}\left(\zeta^{-2} \bar{\Psi}_{L} \Psi_{R}\right)
\end{aligned}
$$

where $q_{\mathrm{U}(1)}$ is the conserved $\mathrm{U}(1)$ charge,

$$
\begin{aligned}
q_{\mathrm{U}(1)} & \equiv \int d z \mathcal{J}_{\mathrm{U}(1)}^{0}, \\
\mathcal{J}_{\mathrm{U}(1)}^{\mu} & =G\left(\bar{w} i \stackrel{\leftrightarrow}{\partial}{ }^{\mu} w+\bar{\Psi} \gamma^{\mu} \Psi-2 \frac{w \bar{w}}{\zeta} \bar{\Psi} \gamma^{\mu} \Psi\right),
\end{aligned}
$$


and

$$
h=-\frac{2}{g_{0}^{2}} \frac{1}{\zeta}
$$

where $g_{0}^{2}$ is the bare coupling constant of the $C P^{1}$ model (i.e. normalized at an ultraviolet scale $M_{U V}$ ). The supercharges are normalized in such a way (see Eq. (4.26)) that the kink mass equals the absolute value of the expectation value of the righthand side. If $\mu$ is real,

$$
q_{\mathrm{U}(1)}=\frac{\theta_{\mathrm{eff}}}{2 \pi}
$$

and, quasiclassically,

$$
M_{\mathrm{kink}}=\left|\mu\left(\frac{2}{g_{0}^{2}}-\frac{1}{2 \pi} \ln \frac{M_{U V}^{2}}{\mu^{2}}+i \frac{\theta_{\mathrm{eff}}}{2 \pi}\right)\right| .
$$

At $\theta_{\text {eff }}=0$ Eqs. (5.26) and (5.15) are identical.

\subsubsection{Kinks in the quasiclassical limit}

In the quasiclassical limit (5.2) the physical meaning of the kink is absolutely transparent. It is the tunneling trajectory from the north pole (the $(1,0)$-string) to the south pole (the $(0,1)$-string). The BPS equation in the holomorphic representation reads

$$
\partial_{z} \bar{w}=-\mu \bar{w} \quad \text { or } \quad \partial_{z} w=-\mu w,
$$

to be compared with Eq. (5.11). Here $\mu$ is assumed real and positive, see Sect. 5.3.1.

The BPS equation (5.27) has a number peculiarities the most important of which is its complexification, i.e. the fact that Eq. (5.27) is holomorphic in $w$. The solution of this equation is, of course, trivial, and can be written as

$$
w(z)=e^{-\mu\left(z-z_{0}\right)-i \alpha} .
$$

Here $z_{0}$ is the kink center while $\alpha$ is an arbitrary phase. In fact, these two parameters enter only in the combination $\mu z_{0}-i \alpha$. As was noted, the notion of the kink center gets complexified. The physical meaning of the modulus $\alpha$ is obvious: there is a continuous family of solitons interpolating between the north and south poles of the target space sphere. This is due to U(1) symmetry. The soliton trajectory can follow

any meridian (Fig. 9). As we will see shortly, there are two fermion counterparts of $z_{0}$ and $\alpha$, which will be referred to as $\eta$ and $\bar{\eta}$.

\subsubsection{Quantization of the bosonic moduli}

To carry out conventional quasiclassical quantization we, as usual, assume the moduli $z_{0}$ and $\alpha$ in Eq. (5.28) to be (weakly) time-dependent, substitute (5.28) in the 


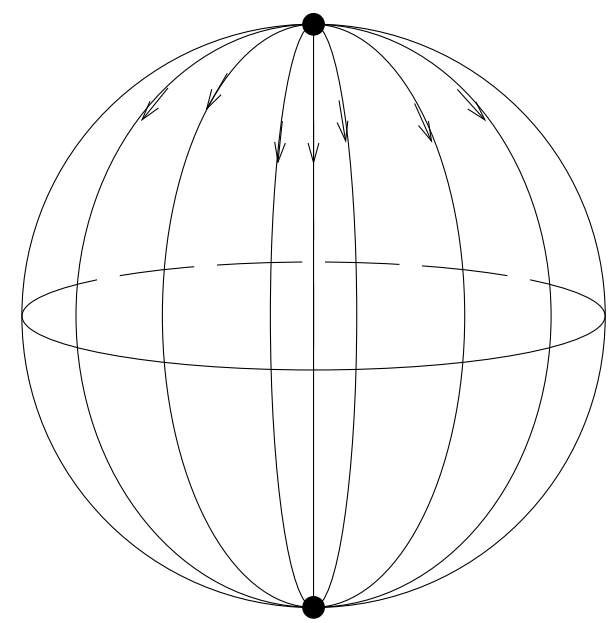

Figure 9: The soliton solution family. The collective coordinate $\alpha$ in Eq. (5.28) spans the interval $0 \leq \alpha \leq 2 \pi$. For given $\alpha$ the soliton trajectory on the target space sphere follows a meridian, so that when $\alpha$ varies from 0 to $2 \pi$ all meridians are covered.

bosonic Lagrangian (4.36), integrate over $z$ and thus derive a quantum-mechanical Lagrangian describing moduli dynamics. In this way we obtain

$$
\mathcal{L}_{\mathrm{QM}}=-M_{\mathrm{kink}}+\frac{M_{\mathrm{kink}}}{2} \dot{z}_{0}^{2}+\frac{\beta}{\mu} \dot{\alpha}^{2}-\frac{\theta_{\mathrm{eff}}}{2 \pi} \dot{\alpha}
$$

The variable $\alpha$ is compact. The canonic momentum $\pi_{\alpha}$ conjugate to $\alpha$ must be defined as

$$
\pi_{\alpha}=\frac{2 \beta}{\mu} \dot{\alpha}-\frac{\theta_{\mathrm{eff}}}{2 \pi} \longrightarrow-i \frac{d}{d \alpha}
$$

With these definitions

$$
q_{\mathrm{U}(1)}=\frac{\theta_{\mathrm{eff}}}{2 \pi}+\pi_{\alpha}
$$

while the Hamiltonian

$$
H=M_{\mathrm{kink}}+\frac{\mu}{4 \beta}\left(\frac{\theta_{\mathrm{eff}}}{2 \pi}\right)^{2}+O\left(\pi_{\alpha}, \pi_{\alpha}^{2}\right) .
$$

For the BPS state $\pi_{\alpha}=0$, and Eqs. (5.31) and (5.32) are consistent with Eq. (5.26). In fact, they describe a whole tower of BPS kinks since $\theta_{\text {eff }}$ is defined modulo $2 \pi k$ with integer $k$. The states with $q_{\mathrm{U}(1)} \neq 0$ are "dyonic" kinks. They have a nonvanishing charge with respect to the global $\mathrm{U}(1)$ symmetry present in the $\mathrm{CP}(1)$ model with the twisted mass. 


\subsubsection{Switching on fermion moduli}

The equations for the fermion zero modes of the kink are

$$
\begin{aligned}
& \partial_{z} \Psi_{L}-\frac{2}{\zeta}\left(\bar{w} \partial_{z} w\right) \Psi_{L}+\frac{1-\bar{w} w}{\zeta} \mu \Psi_{R}=0, \\
& \partial_{z} \Psi_{R}-\frac{2}{\zeta}\left(\bar{w} \partial_{z} w\right) \Psi_{R}-\frac{1-\bar{w} w}{\zeta} \mu \Psi_{L}=0,
\end{aligned}
$$

plus similar equations for $\bar{\Psi}$. It is not difficult to find normalizable solutions to these equations, either directly, or using supersymmetry,

$$
\left(\begin{array}{l}
\Psi_{R} \\
\Psi_{L}
\end{array}\right)=\eta\left(\frac{\mu}{2 \beta}\right)^{1 / 2}\left(\begin{array}{c}
-i \\
1
\end{array}\right) e^{-\mu\left(z-z_{0}\right)}
$$

and

$$
\left(\begin{array}{l}
\bar{\Psi}_{R} \\
\bar{\Psi}_{L}
\end{array}\right)=\bar{\eta}\left(\frac{\mu}{2 \beta}\right)^{1 / 2}\left(\begin{array}{l}
i \\
1
\end{array}\right) e^{-\mu\left(z-z_{0}\right)},
$$

where the numerical prefactors are introduced to ensure proper normalization of the quantum-mechanical Lagrangian.

Now, to perform quasiclassical quantization the fermion moduli $\eta, \bar{\eta}$ are assumed to be time-dependent, and we derive their quantum mechanics starting from the original Lagrangian (4.18), (4.37),

$$
\mathcal{L}_{\mathrm{QM}}^{\prime}=i \bar{\eta} \dot{\eta}
$$

implying the anticommutation relations

$$
\{\bar{\eta} \eta\}=1, \quad\{\bar{\eta} \bar{\eta}\}=0, \quad\{\eta \eta\}=0
$$

which tell us that the wave function is two-component (i.e. the kink supermultiplet is two-dimensional). One can implement Eq. (5.37) by choosing e.g. $\bar{\eta}=\sigma^{+}, \eta=\sigma^{-}$. The eigenstates then will be of the type $|\uparrow\rangle$ and $|\downarrow\rangle$.

Upon quantization of the fermion moduli one finds that the $U(1)$ charge of the BPS kink states gets a fractional shift,

$$
\frac{\theta_{\mathrm{eff}}}{2 \pi} \rightarrow \frac{\theta_{\mathrm{eff}}}{2 \pi} \pm \frac{1}{2}
$$

where the plus or minus signs correspond to $|\uparrow\rangle$ and $|\downarrow\rangle$, respectively. This fractional shift is due to fact that there are two fermion zero modes, and is conceptually similar to the well-known charge fractionalization phenomenon [43]. 


\subsubsection{Exact solution}

The above features of the BPS kinks in the $C P^{1}$ model are concisely summarized by the exact expression for the corresponding central charge (cf. Eq. (5.22))

$$
Z_{2 D}=i \mu q+\mu_{D} T
$$

obtained in Ref. [4] exploiting methods similar to those of Seiberg and Witten [11]. Here $T$ is the topological charge of the kink under consideration. In this work

$$
T=1
$$

while the parameter $q$ in Eq. (5.39)

$$
q=0, \pm 1, \pm 2, \ldots
$$

The quantity $\mu_{D}$ is introduced in analogy with $a_{D}$ of Ref. [11],

$$
\mu_{D}=\frac{\mu}{\pi}\left[\frac{1}{2} \ln \frac{\mu+\sqrt{\mu^{2}+4 \Lambda_{C P(1)}^{2} e^{-i \theta}}}{\mu-\sqrt{\mu^{2}+4 \Lambda_{C P(1)}^{2} e^{-i \theta}}}-\sqrt{1+\frac{4 \Lambda_{C P(1)}^{2} e^{-i \theta}}{\mu^{2}}}\right]
$$

where $\mu$ is now assumed to be complex, as in Eq. (5.20). The two-dimensional central charge is normalized in such a way that $M_{\text {kink }}=\left|Z_{2 D}\right|$.

Note that the integer parameter $q$ in Eq. (5.39) is not the physical U(1) charge. The latter is related to $q$ is follows (at $T=1$ ):

$$
q_{\mathrm{U}(1)}=q+\operatorname{Im} \frac{\mu_{D}}{\mu} .
$$

The limit $|\mu| / \Lambda_{C P(1)} \rightarrow \infty$ corresponds to the quasiclassical domain, while corrections of the type $\left(\Lambda_{C P(1)} / \mu\right)^{2 k}$ are induced by instantons.

\subsubsection{Multiplicity}

The two-dimensional $C P^{1}$ model has $\mathcal{N}=2$ supersymmetry; correspondingly, each shortened supermultiplet is two-dimensional. In fact, one can introduce a fermion parity [20], and each shortened supermultiplet has one plus and one minus state with respect to this parity. The question to be addressed below is whether one has an extra degeneracy, and - if yes - how many degenerate two-dimensional supermultiplets one has for given values of parameters. We will limit ourselves to $T=1$, but the value of $q_{\mathrm{U}(1)}$ can be arbitrary.

Let us start from the quasiclassical limit $\mu \gg \Lambda_{C P(1)}$ assuming $\mu$ to be real. Of course, at $\mu \neq 0$, the results are $\theta$-dependent. Let us consider a general case, $\theta \neq 0$. Then, for each value of the soliton mass determined from Eqs. (5.39) and (5.42) we 
have one two-dimensional supermultiplet. There is a whole tower of $T=1$ solitons corresponding to $q=k$. In this tower a single two-dimensional supermultiplet is the lightest.

Now, if $\theta=0$ (or $\pm 2 \pi, \pm 4 \pi$, etc.), we get a a special case, because in this case the states with distinct $k$ conspire. For each given value of the mass from Eqs. (5.39) and (5.42) we have two degenerate two-dimensional supermultiplets.

One can readily rephrase these statements allowing oneself to travel in the complex $\mu$ plane. The above degeneracy will hold provided $\theta_{\text {eff }}=0$.

What happens when one travels from the domain of large $|\mu|$ to that of small $|\mu|$ ? If $\mu=0$ we know e.g. from the mirror representation (4.39), that there are two degenerate two-dimensional supermultiplets, corresponding to the CFIV index $=2$. Of course, at $\mu=0$ there is no $\theta$ dependence, and two BPS supermultiplets - those with $\{q, T\}$ charges $(0,1)$ and $(1,1)$ - are degenerate. Away from the point $\mu=0$ the masses of these states are no longer equal; there are two singular points with one of the two states becoming massless at each. The region containing the point $\mu=0$ is separated from the quasiclassical region of large $\mu$ by an infinite family of curves of the marginal stability (CMS) on which the infinite number of other BPS states, visible quasiclassically, decay. Thus, the infinite tower of the $\{q, T\}$ BPS states existing in the quasiclassical domain degenerates in just two stable BPS states in the vicinity of $\mu=0$.

The CFIV index for the BPS states in question is independent of $D$ and $F$ terms but does depend, generally speaking, on twisted $F$ terms, and the twisted mass parameter $\mu$, in particular. The CFIV index can change discontinuously as one crosses a CMS. For more details see e.g. the last work in Ref. [41] (in particular, Eq. (2.9)) although this work does not allow for the possibility of a global Noether charge such as $q_{\mathrm{U}(1)}$.

In the four-dimensional $\mathcal{N}=2$ SUSY theories on the Coulomb branch it turned out possible [44] to find all CMS explicitly by a careful study of the phase of the exact central charges. Since the latter are fully equivalent [4] to a central charge in the two-dimensional sigma model, a similar analysis should go through in the $C P^{1}$ model as well, see also Sect. 7 .

\subsection{Implications for confined monopoles}

Since, as we have proven, the $C P^{1}$ model presents the macroscopic description of solitons in our four-dimensional microscopic model, all results regarding the BPS kinks summarized in Sect. 5.3 can be immediately translated in statements regarding the confined monopoles $-1 / 4$ BPS states in $\mathcal{N}=2$ four-dimensional Yang-Mills theory.

If $|\Delta m| \gg \Lambda$, our microscopic model has an infinite tower of "dyonic" confined monopoles. In addition to the topological charge $T$ these BPS states carry a 
Noether U(1) charge. This $\mathrm{U}(1)$ charge has nothing to do with the electric charge of the Julia-Zee dyons [45]. The latter is associated with the gauge $\mathrm{U}(1)$ symmetry which remains unbroken in the 't Hooft-Polyakov theory. In our theory the gauge symmetry is completely broken; there are no long-range forces. It is a global $\mathrm{U}(1)$ symmetry that survives. The "dyonic" confined monopoles are charged with respect to this global U(1). Their masses are given by Eqs. (5.39), (5.42), with $T=1$ and $q=k$, see also (5.43). Generally speaking, the "dyonic" confined monopoles carry irrational $\mathrm{U}(1)$ charges. Thus, in the presence of the $\theta$ term, they experience the same charge "irrationalization" [46] as the 't Hooft-Polyakov monopoles (the Witten phenomenon). ${ }^{15}$

Needless to say, the dyonic confined monopoles do not exist without the nonAbelian strings attached to them. The latter carry a non-Abelian magnetic flux. The elementary excitations of these strings carry integer $q_{\mathrm{U}(1)} \neq 0$ but $T=0$. As $|\Delta m|$ decreases, the dyonic confined monopoles become unstable, as one passes through a family of CMS. Eventually, only two monopole supermultiplets survive as stable $1 / 4$ BPS saturated states. At $\Delta m=0$ they are degenerate, which reflects the global $\mathrm{SU}(2)$ symmetry of the microscopic model.

At $\Delta m \neq 0$ the nontopological (i.e. $T=0$ and $q_{\mathrm{U}(1)}= \pm 1$ ) excitations of the string are BPS states with mass $\mu$ confined to the string. They can be interpreted as follows. Inside the string the squark profiles vanish, effectively bringing us towards the Coulomb branch $(\xi=0)$ where the $W$ bosons and quarks would become BPS saturated states in the bulk. As a matter of fact, on the Coulomb branch the $W$ boson and off-diagonal quark mass would just equal $\mu$. Hence, the $T=0$ BPS excitation of the string is a wave of such $W$ bosons/quarks propagating along the string. One could call it a "confined $W$ boson/quark." It is localized in the perpendicular but not in the transverse direction. What is important, it has no connection with the bulk Higgs phase $W$ bosons which are not BPS and much heavier than $\mu$. Neither these nontopological excitations have connection with the bulk quarks in our microscopic model which are not BPS saturated too.

\section{Anomaly: matching the central charges}

If $\mu=0$, which will be assumed in this section, the $C P^{1}$ model runs into strong coupling and its physics is determined by quantum effects. In particular, at $\mu=0$ the vacuum expectation value $\left\langle a^{3}\right\rangle=0$; the strings become genuinely non-Abelian.

There are no massless states in the $C P^{1}$ model at $\mu=0$. In particular, the kink mass is of the order of $\Lambda_{C P(1)}$, as it is clear e.g. from the mirror description of the model (4.39). On the other hand, in this limit both the last term in (5.4) and the surface term in (5.12) vanish for the monopole and kink masses, respectively.

\footnotetext{
${ }^{15}$ For semi-integer $q_{\mathrm{U}(1)}$ there is an additional mass degeneracy.
} 
This puzzle is solved by the following observation: anomalous terms in the central charges of both four-dimensional and two-dimensional SUSY algebras emerge. Below we discuss the relation between the central charge anomalies in the microscopic and macroscopic theories.

In the microscopic theory the central charge associated with the monopole has the following general form:

$$
\left\{Q_{\alpha}^{f} Q_{\beta}^{g}\right\}=\varepsilon_{\alpha \beta} \varepsilon^{f g} 2 Z_{4 D},
$$

where $Z_{4 D}$ is an $\mathrm{SU}(2)_{R}$ singlet. It is most convenient to write $Z_{4 D}$ as a topological charge (i.e. the integral over a topological density),

$$
Z_{4 D}=\int d^{3} x \zeta^{0}(x)
$$

In the model at hand

$$
\begin{aligned}
\zeta^{\mu} & =\frac{1}{2} \varepsilon^{\mu \nu \rho \sigma} \partial_{\nu}\left(\frac{i}{g_{2}^{2}} a^{a} F_{\rho \sigma}^{a}+\frac{i}{g_{1}^{2}} a F_{\rho \sigma}\right. \\
& \left.+\frac{c}{4 \pi^{2}}\left[\lambda_{f \alpha}^{a}\left(\sigma_{\rho}\right)^{\alpha \dot{\alpha}}\left(\bar{\sigma}_{\sigma}\right)_{\dot{\alpha} \beta} \lambda^{a f \beta}+2 g_{2}^{2} \tilde{\psi}_{A \alpha}\left(\sigma_{\rho}\right)^{\alpha \dot{\alpha}}\left(\bar{\sigma}_{\sigma}\right)_{\dot{\alpha} \beta} \psi^{A \beta}\right]\right),
\end{aligned}
$$

where $c$ is a numerical coefficient which can be obtained from a one-loop calculation in the $\mathcal{N}=2$ regularized $\mathrm{SU}(2) \times \mathrm{U}(1)$ gauge theory which is our microscopic model. The operator in the square brackets represents the anomaly, since $c$ vanishes at the tree level. Note that the general structure of the operator in the square brackets is unambiguously fixed by dimensional arguments, the Lorentz symmetry and other symmetries of the microscopic theory. It is only the coefficient $c$ which is unknown. The anomalous term plays a crucial role in the Higgs phase. ${ }^{16}$

It is quite difficult to calculate $c$ directly because to this end one needs an explicit $\mathcal{N}=2$ ultraviolet regularization of the four-dimensional theory. Although this is doable, the direct calculation has not been completed yet. However, one can find $c$ indirectly, by comparing the expressions for the masses of the BPS saturated confined monopole on the one hand, and the BPS saturated kink in the $C P(1)$ on the other hand. More precisely, we will compare the corresponding central charges in the microscopic and macroscopic theories.

The mass of the monopole in terms of the central charge is given by

$$
M_{m}=\sqrt{2}\left|Z_{4 D}\right|
$$

\footnotetext{
${ }^{16} \mathrm{~A}$ similar (albeit distinct) effect exists on the Coulomb branch. The relationship between the 't Hooft-Polyakov monopole mass and the $\mathcal{N}=2$ central charge is analyzed in the recent publication [21], which identifies an anomaly in the central charge explaining a constant (i.e. nonlogarithmic) term in the monopole mass on the Coulomb branch. The result of Ref. [21] is in agreement with the Seiberg-Witten formula for the monopole mass.
} 
In the limit $\mu=0$ the classical term in $\left|Z_{4 D}\right|$ vanishes, and the central charge is determined by the last anomalous term in Eq. (6.3). Now our task is to project it onto the macroscopic theory. Substituting the superorientational fermion zero modes of the string (4.49) in the square brackets in Eq. (6.3) we get

$$
\begin{aligned}
Z_{4 D} & =-\frac{c}{2 \pi} \int d z \partial_{z}\left(\chi_{1}^{a} \chi_{2}^{a}-i \varepsilon^{a b c} n^{a} \chi_{1}^{b} \chi_{2}^{c}\right) \\
& \times \int_{0}^{\infty} r d r\left\{\left(\frac{d}{d r} \rho(r)\right)^{2}+\frac{1}{r^{2}} f_{3}^{2}(1-\rho)^{2}\right. \\
& \left.+g_{2}^{2}\left[\frac{1}{2} \rho^{2}\left(\phi_{1}^{2}+\phi_{2}^{2}\right)+(1-\rho)\left(\phi_{1}-\phi_{2}\right)^{2}\right]\right\} .
\end{aligned}
$$

Here we recognize the same normalization integral which emerges in Sect. 4.1. Keeping in mind that $I=1$ we arrive at

$$
Z_{4 D}=-\frac{c}{2 \pi} \int d z \partial_{z}\left(\chi_{1}^{a} \chi_{2}^{a}-i \varepsilon^{a b c} n^{a} \chi_{1}^{b} \chi_{2}^{c}\right)
$$

Rewriting the bifermion operator in (6.6) in the holomorphic representation [35],

$$
\chi_{1}^{a} \chi_{2}^{a}-i \varepsilon^{a b c} n^{a} \chi_{1}^{b} \chi_{2}^{c}=-\frac{4}{\left(1+|w|^{2}\right)^{2}} \bar{\Psi}_{L} \Psi_{R},
$$

and substituting it in (6.6) we finally get

$$
Z_{4 D}=\frac{2 c}{\pi} \int d z \partial_{z}\left(\frac{1}{\zeta^{2}} \bar{\Psi}_{L} \Psi_{R}\right) .
$$

The right-hand side of this equation reproduces the two-dimensional anomaly (4.26) provided that the coefficient

$$
c=\frac{1}{2 \sqrt{2}} .
$$

The four-dimensional central charge (6.8) with the coefficient $c$ given by (6.9) leads to the result (5.19) for the monopole mass in the limit $\Delta m=0$, provided we use the values of the fermion condensate (4.27) in the two vacua (at $z= \pm \infty$ ) of the $C P^{1}$ model.

\section{2D sigma-model kink and 4D Seiberg-Witten exact solution}

Why the 't Hooft-Polyakov monopole mass (i.e. on the Coulomb branch at $\xi=0$ ) is given by the same formula (5.7) as the mass (5.14) of the strongly confined large- $\xi$ 
monopole (subject to condition (5.2))? This fact was noted in Sect. 5.2. Now we will explain the reason lying behind this observation. En route, we will explain another striking observation made in Ref. [4]. A remarkably close parallel between the fourdimensional Yang-Mills theory with $N_{f}=2$ and the two-dimensional $C P^{1}$ model was noted, at an observational level, by virtue of comparison of the corresponding central charges. The observation was made on the Coulomb branch of the SeibergWitten theory, with unconfined 't Hooft-Polyakov-like monopoles/dyons. Valuable as it is, the parallel was quite puzzling since the solution of the $C P^{1}$ model seemed to have no physics connection to the Seiberg-Witten solution. The latter gives the mass of the unconfined monopole in the Coulomb phase at $\xi=0$ while the $C P^{1}$ model emerges only in the Higgs phase of the microscopic theory.

Physics lying behind the above remarkable parallel will be revealed here. First and foremost, the previous study revealed the fact that the $C P^{1}$ model is a macroscopic description of the four-dimensional Yang-Mills theory with $N_{f}=2$ in the Higgs phase. This establishes a direct correspondence between the $C P^{1}$ model and our microscopic model at $|\mu| \ll \xi$. Needless to say, the correspondence covers the central charges, CMS, dyonic excitations, and all other features of the two theories in $4 \mathrm{D}$ and $2 \mathrm{D}$, respectively.

Now we will show, that in the BPS sector (and only in this sector) the correspondence extends further, since the parameter $\xi$, in fact, cannot enter relevant formulae. Therefore, one can vary $\xi$ at will, in particular, making it less than $|\mu|$ or even tending to zero, where $C P^{1}$ is no more the macroscopic model for our microscopic theory. Nevertheless, the parallel expressions for the central charges and other BPS data in 4D and 2D, trivially established at $|\mu| \ll \xi$, will continue to hold even on the Coulomb branch. The "strange coincidence" we observed in Sect. 5.2 is no accident. We deal here with an exact relation which stays valid including both perturbative and nonperturbative corrections.

Physically the monopole in the Coulomb phase is very different from the one in the confinement phase, see Fig. 1. In the Coulomb phase it is a 't Hooft-Polyakov monopole, while in the confinement phase it becomes related to a junction of two non-Abelian strings. Still let us show that the masses of these two objects are given by the same expression,

$$
M_{m}^{\text {Coulomb }}=M_{m}^{\text {confinement }},
$$

provided that $\Delta m$ and the gauge couplings are kept fixed. The superscripts refer to the Coulomb and monopole-confining phases, respectively.

Our point is that the mass of the monopole cannot depend on the FI parameter $\xi$. Start from the monopole in the Coulomb phase at $\xi=0$. Its mass is given by the exact Seiberg-Witten formula [12]

$$
M_{m}^{\text {Coulomb }}=\sqrt{2}\left|a_{D}^{3}\left(a^{3}=-\frac{\mu}{\sqrt{2}}\right)\right|=\left|\frac{\mu}{\pi} \ln \frac{\mu}{\Lambda}+\mu \sum_{k=1}^{\infty} c_{k}\left(\frac{\Lambda}{\mu}\right)^{2 k}\right|,
$$




\begin{tabular}{|c|c|c|c|c|c|c|c|c|}
\hline Field/parameter & $a$ & $a^{a}$ & $\lambda^{\alpha}$ & $q$ & $\psi^{\alpha}$ & $m_{A}$ & $\Lambda$ & $\xi$ \\
\hline $\mathrm{U}(1)_{R}$ charge & 2 & 2 & 1 & 0 & -1 & 2 & 2 & 0 \\
\hline
\end{tabular}

Table 2: The $\mathrm{U}(1)_{R}$ charges of fields and parameters of the microscopic theory.

where $a_{D}^{3}$ is the dual Seiberg-Witten potential for the $\mathrm{SU}(2)$ gauge subgroup, and we take into account that for $N_{f}=2$ the first coefficient of the $\beta$ function is 2 . Here $a^{3}=-\mu / \sqrt{2}$ is the argument of $a_{D}^{3}$, the logarithmic term takes into account the one-loop result (3.8) for the $\mathrm{SU}(2)$ gauge coupling at the scale $\mu$, while the power series is the expansion in instanton-induced corrections.

Now, if we introduce a small FI parameter $\xi \neq 0$ in the theory, on dimensional grounds, we could expect in Eq. (7.2) corrections to the monopole mass in powers of $\sqrt{\xi} / \Lambda$ and/or $\sqrt{\xi} / \mu$. These corrections are forbidden by the $\mathrm{U}(1)_{R}$ charges. Namely, the $\mathrm{U}(1)_{R}$ charges of $\Lambda$ and $\mu=\Delta m$ are equal to 2 (and so is the $\mathrm{U}(1)_{R}$ charge of the central charge under consideration) while $\xi$ has a vanishing $\mathrm{U}(1)_{R}$ charge. For convenience, the $\mathrm{U}(1)_{R}$ charges of different fields and parameters of the microscopic theory are collected in Table 2 . Thus, neither $(\sqrt{\xi} / \Lambda)^{k}$ nor $(\sqrt{\xi} / \mu)^{k}$ can appear.

By the same token, we could start from the confined monopole at large $\xi$, and study the dependence of the monopole (string junction) mass as a function of $\xi$ as we reduce $\xi$. Again, the above arguments based on the $\mathrm{U}(1)_{R}$ charges tell us that corrections in powers of $\Lambda / \sqrt{\xi}$ and $\mu / \sqrt{\xi}$ cannot appear. This leads us to Eq. (7.1).

Another way to arrive at the same conclusion is to observe that the mass of the monopole is determined by the central charge (6.2). This central charge is a holomorphic quantity and, thus, cannot depend on the FI parameter $\xi$ which is not holomorphic (it is a component of the $\mathrm{SU}(2)_{R}$ triplet [23]).

Now recall that the mass of the monopole in the confinement phase is given by the kink mass in the $\mathcal{N}=2 C P^{1}$ model, see (5.17). Thus, we obtain

$$
M_{m}^{\text {Coulomb }} \leftrightarrow M_{m}^{\text {confinement }} \leftrightarrow M_{\text {kink }}
$$

In particular, at the one-loop level, the kink mass is given by Eq. (5.15). This leads to the relation $\Lambda_{2 D}=\Lambda$ between the $2 \mathrm{D}$ and $4 \mathrm{D}$ dynamical scales which we noted earlier as a "strange coincidence," see Eq. (4.23). Now we know the physical reason behind it. A puzzling question immediately coming to one's mind is what would happen with more quark flavors. One may suspect that adding extra quark flavors in our microscopic theory (i.e. more than two) will change its renormalization-group flow while the renormalization-group flow in the macroscopic model seemingly remains the same, which would certainly destroy the correspondence. This conclusion is wrong because, with more than two flavors in the microscopic theory, the strings 
that form in the microscopic theory become semilocal [47]. Semilocal strings has additional zero modes associated with the change in their transverse size. Thus, the moduli space of these strings changes, and is no longer given by the $C P^{1}$ model [5]. The macroscopic model is just different.

Summarizing, the exact expression for the BPS kink masses in the 2D sigma model is given by $\left|Z_{2 D}\right|$, see Eqs. (5.39) and (5.42). These are also the expressions for the confined monopoles (and dyonic monopoles) in the 4D Yang-Mills theory, and, in view of the above, the expressions coinciding with the SU(2) SeibergWitten monopole/dyon solution on the Coulomb branch at the particular point $a^{3}=-\mu / \sqrt{2}$. Although we do not discuss it in the present paper, the above relation can be generalized (cf. [4, 48]) to theories with the $\mathrm{SU}(N) \times \mathrm{U}(1)$ gauge group and $N_{f}=N$ flavors on the four-dimensional side, and $C P^{(N-1)}$ sigma models on the twodimensional side. This is because the effective world-sheet theory for non-Abelian strings in $\mathcal{N}=2$ QCD with the $\mathrm{SU}(N) \times \mathrm{U}(1)$ gauge group and $N_{f}=N$ flavors is the $C P^{(N-1)}$ sigma model $[5,3]$. We are planning to return to this issue elsewhere.

\section{Conclusions}

In this paper we studied various dynamical regimes associated with the confined monopoles that occur on the Higgs branch of $\mathcal{N}=2$ two-flavor QCD. The focus of our consideration is the quasiclassical treatment of the string junctions in the domain

$$
\Lambda \ll|\Delta m| \ll \sqrt{\xi} .
$$

The BPS sector is fully solvable. The confined monopole carries two elementary strings attached to it, and can be viewed as a string junction. We derived a complete set of the first-order master equations, and found their solutions corresponding to $1 / 2$ and $1 / 4$ BPS saturation. The string junction is $1 / 4$ BPS. We obtained the orientational and superorientational zero modes, introduced the corresponding moduli (quasi-moduli) and developed a macroscopic description of strings and their junctions based on two-dimensional $C P^{1}$ model with a twisted mass related (equal) to the difference of the mass parameters of two flavors in our microscopic theory. The masses and other characteristics of the confined monopoles are matched with those of the $C P^{1}$-model kinks. The matching reveals, in particular, the occurrence of an anomaly in the monopole central charge in 4D Yang-Mills theory.

Building on the established identification of the microscopic and microscopic models, we expand in two crucial directions. We study what becomes of the confined monopole in the bona fide non-Abelian limit $\Delta m \rightarrow 0$ where the global SU(2) symmetry is restored. To this end we considered such monopoles (classically they would become massless and infinitely spread) as quantum states interpolating between two vacua of the $C P^{1}$ model with vanishing twisted mass. This is a highly 
quantum regime, whose solution is known, however, e.g. from the mirror description of the $C P^{1}$ model. The classical would-be explosion never happens. Instead, the monopole becomes stabilized by nonperturbative dynamics in the effective 2D sigma model on the string world sheet. This monopole, aka the $C P^{1}$-model kink, acquires a nonvanishing mass of the order of $\Lambda$ and a finite size of the order of $\Lambda^{-1}$.

Another direction is the small- $\xi$ domain. If $\Delta m$ is kept fixed, while $\xi$ decreases, we move towards a weaker confinement, eventually ending up on the Coulomb branch (i.e. $\xi=0$ ) where the Seiberg-Witten exact solution applies. Needless to say, in this limit the $C P^{1}$ model is irrelevant to the macroscopic description. In the Coulomb phase the $\mathrm{SU}(2)$ gauge subgroup gets broken down to $\mathrm{U}(1)$ [11], but the residual $\mathrm{U}(1)$ is not broken if $\mathcal{N}=2$ is maintained. The breaking of $\mathrm{U}(1)$ occurs only if an explicit breaking of $\mathcal{N}=2$ is introduced "by hand," and strings which develop in this case are Abelian (Abrikosov-Nielsen-Olesen) strings. The monopole never becomes bona fide non-Abelian, although it acquires a mass given by the SeibergWitten formula. Physics of the Coulomb phase has nothing to do with that of the $C P^{1}$ model. And, nevertheless, as was observed in Ref. [4], the BPS spectrum of the $C P^{1}$ model is in one-to-one correspondence with the exact Seiberg-Witten solution.

The puzzle is solved by the following observation. Physics of our microscopic model in the Higgs phase, $\xi \neq 0$, is perfectly similar to that of the $C P^{1}$ model. More precisely, the BPS sectors can be mapped one onto another. On the other hand, holomorphic nature of the central charges preclude them from developing a $\xi$ dependence. Therefore, BPS data in the Higgs phase are related to those in the Coulomb phase.

Needless to say, of more practical interest is condensation of non-Abelian monopoles which must be responsible for non-Abelian confinement of quarks - a phenomenon dual to the one studied in this paper, where confined are non-Abelian monopoles, while quarks condense. We are not sure how to dualize our results. One way to approach this problem is a reduction of the quark mass parameters $m_{1,2}$. If they get sufficiently small, below the Argyres-Douglas point [49], the quarks acquire magnetic quantum numbers [44] and their condensation should trigger confinement of color-electric charges.

\section{Acknowledgments}

We are grateful to Alexander Gorsky, Adam Ritz and Arkady Vainshtein for very useful discussions. We would like to thank N. Dorey for extremely valuable communications. The work of M.S. is supported in part by DOE grant DE-FG02-94ER408. A. Y. is supported in part by the Russian Foundation for Basic Research grant No. 02-02-17115, by INTAS grant No. 00-00334 and by Theoretical Physics Institute at the University of Minnesota. 


\section{Note added (March 13, 2004)}

David Tong has just informed us that he is finalizing a paper, a sequel to his inspiring publication [6], which has an overlap with the results reported here.

\section{Note added (May 22, 2004)}

After this paper was submitted (and accepted) for publication in Physical Review $\mathrm{D}$ we became aware of some new circumstances. First, string junctions in the YangMills-Higgs system were considered (in a nonsupersymmetric context) in the 1980's. In a model with the $\mathrm{SU}(2) \rightarrow \mathrm{U}(1) \rightarrow Z_{2}$ symmetry breaking pattern it was found [50] that there exist two distinct (degenerate) strings, each carrying $2 \pi / e$ units of the magnetic flux. Although these strings had no orientational moduli, they did support kinks interpolating between them, which were called "monopole beads" by the authors. Topological arguments were presented [50] proving the stability of such field configurations. Further analysis was carried out in Ref. [51] where it was shown that, in fact, many popular models of the type $G \rightarrow H \rightarrow Z_{2}$ do not support stable monopole beads. Non-translational zero modes of strings were discussed in a $\mathrm{U}(1) \times \mathrm{U}(1)$ model $[52,53]$, and later, in more contrived models, in Ref. [54] (the latter paper is entitled "Zero Modes Of Non-Abelian Vortices").

It is worth emphasizing that, along with apparent similarities of which we will say later, there are drastic distinctions between the "non-Abelian strings" we consider here and the strings that were discussed in the 1980's. In particular, in the example treated in Ref. [54] the gauge group is not completely broken in the vacuum, and, therefore, there are massless gauge fields in the bulk. If the unbroken generator acts nontrivially on the string flux (which is proportional to a broken generator) then it can and does create zero modes. Some divergence problems ensue.

In contrast, in our case the gauge group is completely broken (up to a discrete subgroup $Z_{2}$ ). The theory in the bulk is fully Higgsed. The unbroken group $\mathrm{SU}(2)_{C+F}$, a combination of the gauge and flavor groups, is global. There are no massless fields in the bulk.

We could model the example considered in [54] if we gauge our unbroken global symmetry $\mathrm{SU}(2)_{C+F}$ with respect to yet another ad hoc gauge field $B_{\mu}$.

Some technical points first introduced in Ref. [54] are close to constructions exploited in our papers $[1,2,27]$ and the present paper. In particular, generation of $k=0,3$ components of the gauge potential upon switching on the $t, z$ dependence of the moduli, determined by an extra profile function $\rho$ (see Eq. (4.1)) was first implemented in [54].

We are very grateful to Mark Hindmarsh for pointing out to us the above publications which, unfortunately, escaped our attention.

We use this opportunity to add that the Hanany-Tong paper mentioned in our Note of March 13, was posted on March 15 [55]. Another relevant development was 
reported in a publication of Isozumi et al. [56] which was posted on May 16. The authors obtained a general solution of $1 / 4$ BPS equations similar to those we have derived and discussed in the bulk of the paper (see also our previous works $[1,2]$ ). Their construction is applicable in the strong coupling limit in $\mathrm{U}(N)$ gauge theories with the number of fundamental hypermultiplets exceeding $N$. In fact, due to this reason, their strings are "semilocal strings." In this case one has Higgs branches instead of isolated vacua, as is the case in our analysis. When one goes to the strong coupling limit the model effectively reduces to a sigma model on the Higgs branch. Strings become instantons lifted to 4D (semilocal strings). In this case solving the first order equations seems to be an easier task than in ours. This paves the way to a full analytical solution. 


\section{References}

[1] M. Shifman and A. Yung, Phys. Rev. D 67, 125007 (2003) [hep-th/0212293].

[2] M. Shifman and A. Yung, Localization of non-Abelian gauge fields on domain walls at weak coupling (D-brane prototypes), hep-th/0312257, (Phys. Rev. D, submitted).

[3] R. Auzzi, S. Bolognesi, J. Evslin, K. Konishi and A. Yung, Nucl. Phys. B 673, 187 (2003) hep-th/0307287.

[4] N. Dorey, JHEP 9811, 005 (1998) [hep-th/9806056].

[5] A. Hanany and D. Tong, JHEP 0307, 037 (2003) [hep-th/0306150].

[6] D. Tong, Monopoles in the Higgs phase, hep-th/0307302.

[7] J. M. Maldacena, Adv. Theor. Math. Phys. 2, 231 (1998) [Int. J. Theor. Phys. 38, 1113 (1999)] [hep-th/9711200]; S. S. Gubser, I. R. Klebanov and A. M. Polyakov, Phys. Lett. B 428, 105 (1998) [hep-th/9802109]; E. Witten, Adv. Theor. Math. Phys. 2, 253 (1998) [hep-th/9802150].

[8] J. Polchinski, Phys. Rev. Lett. 75, 4724 (1995) [hep-th/9510017]; see also the excellent text by J. Polchinski, String Theory, Vols. 1 and 2 (Cambridge University Press, Cambridge, 1998).

[9] G. R. Dvali and M. A. Shifman, Phys. Lett. B 396, 64 (1997) (E) B 407, 452 (1997) [hep-th/9612128].

[10] E. Witten, Nucl. Phys. B 507, 658 (1997) [hep-th/9706109].

[11] N. Seiberg and E. Witten, Nucl. Phys. B426, 19 (1994), (E) B430, 485 (1994) [hep-th/9407087].

[12] N. Seiberg and E. Witten, Nucl. Phys. B431, 484 (1994) [hep-th/9408099].

[13] P. Fayet and J. Iliopoulos, Phys. Lett. B 51, 461 (1974).

[14] P. Argyres, M. Plesser and N. Seiberg, Nucl. Phys. B471, 159 (1996) [hepth/9603042].

[15] G. Carlino, K. Konishi and H. Murayama, Nucl. Phys. B 590, 37 (2000) [hepth/0005076].

[16] A. Marshakov and A. Yung, Nucl. Phys. B 647, 3 (2002) [hep-th/0202172]. 
[17] R. Auzzi, S. Bolognesi, J. Evslin and K. Konishi, Non-Abelian monopoles and vortices that confine them, hep-th/0312233.

[18] E. J. Weinberg, Nucl. Phys. B 167, 500 (1980); Nucl. Phys. B 203, 445 (1982).

[19] G. 't Hooft, Nucl. Phys. B 79, 276 (1974); A. M. Polyakov, Pisma Zh. Eksp. Teor. Fiz. 20, 430 (1974) [JETP Lett. 20, 194 (1974)].

[20] A. Losev and M. Shifman, Phys. Rev. D 68, 045006 (2003) [hep-th/0304003].

[21] A. Rebhan, P. van Nieuwenhuizen and R. Wimmer, A new anomaly in the central charge of the $\mathcal{N}=2$ monopole, hep-th/0401116.

[22] A. Hanany, M. J. Strassler and A. Zaffaroni, Nucl. Phys. B 513, 87 (1998) [hep-th/9707244].

[23] A. I. Vainshtein and A. Yung, Nucl. Phys. B 614, 3 (2001) [hep-th/0012250].

[24] K. Bardakci and M. B. Halpern, Phys. Rev. D 6, 696 (1972).

[25] A. Abrikosov, Sov. Phys. JETP 321442 (1957) [Reprinted in Solitons and Particles, Eds. C. Rebbi and G. Soliani (World Scientific, Singapore, 1984), p. 356];

H. Nielsen and P. Olesen, Nucl. Phys. B61 45 (1973) [Reprinted in Solitons and Particles, Eds. C. Rebbi and G. Soliani (World Scientific, Singapore, 1984), p. 365].

[26] A. Ritz, M. Shifman, and A. Vainshtein, to appear.

[27] M. Shifman and A. Yung, Phys. Rev. D 66, 045012 (2002), [hep-th/0205025].

[28] H. J. de Vega and F. A. Schaposnik, Phys. Rev. Lett. 56, 2564 (1986); Phys. Rev. D 34, 3206 (1986).

[29] J. Heo and T. Vachaspati, Phys. Rev. D 58, 065011 (1998) [hep-ph/9801455].

[30] P. Suranyi, Phys. Lett. B 481, 136 (2000) [hep-lat/9912023].

[31] F. A. Schaposnik and P. Suranyi, Phys. Rev. D 62, 125002 (2000) [hepth/0005109].

[32] M. A. C. Kneipp and P. Brockill, Phys. Rev. D 64, 125012 (2001) [hepth/0104171].

[33] K. Konishi and L. Spanu, Int. J. Mod. Phys. A 18, 249 (2003) [hep-th/0106175]. 
[34] A. Gorsky and M. A. Shifman, Phys. Rev. D 61, 085001 (2000) [hepth/9909015].

[35] V. Novikov, M. Shifman, A. Vainshtein, V. Zakharov, Phys. Reports 116, 103 (1984).

[36] A. M. Polyakov, Phys. Lett. B 59, 79 (1975).

[37] G. R. Dvali and M. A. Shifman, Phys. Lett. B 396, 64 (1997), (E) B 407, 452 (1997) [hep-th/9612128]; A. Kovner, M. A. Shifman and A. Smilga, Phys. Rev. D 56, 7978 (1997) [hep-th/9706089]; B. Chibisov and M. A. Shifman, Phys. Rev. D 56, 7990 (1997), (E) D 58, 109901 (1998) [hep-th/9706141].

[38] E. Witten, Nucl. Phys. B 202, 253 (1982).

[39] L. Alvarez-Gaumé and D. Z. Freedman, Commun. Math. Phys. 91, 87 (1983); S. J. Gates, Nucl. Phys. B 238, 349 (1984); S. J. Gates, C. M. Hull and M. Roček, Nucl. Phys. B 248, 157 (1984).

[40] K. Hori and C. Vafa, Mirror symmetry, hep-th/0002222, unpublished.

[41] S. Cecotti, P. Fendley, K. A. Intriligator and C. Vafa, Nucl. Phys. B 386, 405 (1992) [hep-th/9204102]; P. Fendley and K. A. Intriligator, Nucl. Phys. B 372, 533 (1992) [hep-th/9111014]; S. Cecotti and C. Vafa, Commun. Math. Phys. 158, 569 (1993) [hep-th/9211097].

[42] E. B. Bogomolny, Yad. Fiz. 24, 861 (1976) [Sov. J. Nucl. Phys. 24, 449 (1976), reprinted in Solitons and Particles, Eds. C. Rebbi and G. Soliani (World Scientific, Singapore, 1984), p. 389].

[43] R. Jackiw and C. Rebbi, Phys. Rev. D 13, 3398 (1976) [reprinted in Solitons and Particles, Eds. C. Rebbi and G. Soliani (World Scientific, Singapore, 1984), p. 331].

[44] A. Bilal and F. Ferrari, Nucl. Phys. B 516, 175 (1998) [hep-th/9706145].

[45] B. Julia and A. Zee, Nonabelian Gauge Theory," Phys. Rev. D 11, 2227 (1975); M. K. Prasad and C. M. Sommerfield, Phys. Rev. Lett. 35, 760 (1975).

[46] E. Witten, Phys. Lett. B 86, 283 (1979).

[47] A. Achucarro and T. Vachaspati, Phys. Reports 327, 347 (2000) [hep$\mathrm{ph} / 9904229]$.

[48] A. Hanany and K. Hori, Nucl. Phys. B 513, 119 (1998) [hep-th/9707192]. 
[49] P. C. Argyres and M. R. Douglas, Nucl. Phys. B 448, 93 (1995) [hepth/9505062].

[50] M. Hindmarsh and T. W. B. Kibble, Phys. Rev. Lett. 55, 2398 (1985).

[51] A. E. Everett and M. Aryal, Phys. Rev. Lett. 57, 646 (1986).

[52] E. Witten, Nucl. Phys. B249, 557 (1985)

[53] M. Hindmarsh, Phys. Lett. B 225, 127 (1989).

[54] M. G. Alford, K. Benson, S. R. Coleman, J. March-Russell and F. Wilczek, Nucl. Phys. B 349, 414 (1991).

[55] A. Hanany and D. Tong, JHEP 0404, 066 (2004) [hep-th/0403158].

[56] Y. Isozumi, M. Nitta, K. Ohashi and N. Sakai, All Exact Solutions of a 1/4 Bogomol'nyi-Prasad-Sommerfield Equation, hep-th/0405129. 